\title{
Invisible decays of the heavier Higgs boson in the minimal supersymmetric standard model
}

\author{
B.Ananthanarayan, Jayita Lahiri, P. N. Pandita \\ Centre for High Energy Physics, Indian Institute of Science, Bangalore 560 012, India
}

\begin{abstract}
We consider the possibility that the heavier CP-even Higgs boson $\left(H^{0}\right)$ in the minimal supersymmetric standard model (MSSM) decays invisibly into neutralinos in the light of the recent discovery of the $126 \mathrm{GeV}$ resonance at the CERN Large Hadron Collider (LHC). For this purpose we consider the minimal supersymmetric standard model with universal, non-universal and arbitrary boundary conditions on the supersymmetry breaking gaugino mass parameters at the grand unified scale. Typically, scenarios with universal and nonuniversal gaugino masses do not allow invisible decays of the lightest Higgs boson $\left(h^{0}\right)$, which is identified with the $126 \mathrm{GeV}$ resonance, into the lightest neutralinos in the MSSM. With arbitrary gaugino masses at the grand unified scale such an invisible decay is possible. The second lightest Higgs boson can decay into various invisible final states for a considerable region of the MSSM parameter space with arbitrary gaugino masses as well as with the gaugino masses restricted by universal and nonuniversal boundary conditions at the grand unified scale. The possibility of the second lightest Higgs boson of the MSSM decaying into invisible channels is more likely for arbitrary gaugino masses at the grand unified scale. The heavier Higgs boson decay into lighter particles leads to the intriguing possibility that the entire Higgs boson spectrum of the MSSM may be visible at the LHC even if it decays invisibly, during the searches for an extended Higgs boson sector at the LHC. In such a scenario the nonobservation of the extended Higgs sector of the MSSM may carefully be used to rule out regions of the MSSM parameter space at the LHC.
\end{abstract}

PACS numbers: $12.60 . \mathrm{Jv}, 14.80 . \mathrm{Da}, 14.80 . \mathrm{Ly}, 14.80 . \mathrm{Nb}$

\section{INTRODUCTION}

With the discovery [1-4] of a neutral state around a mass of $126 \mathrm{GeV}$ at the CERN Large Hadron Collider (LHC), a new era has begun in our quest for the understanding of the fundamental constituents of matter and the forces between them. Although, the properties of the discovered state are consistent with the properties of the standard model (SM) Higgs boson, it also opens up a window for searches of new physics. It is well known that the SM Higgs sector suffers from the naturalness and hierarchy problems, thereby rendering a light Higgs boson technically unnatural. The most popular, and well motivated, extension of the SM which renders a light Higgs boson technically natural include supersymmetric models [5], of which the minimal supersymmetric standard model (MSSM) is perhaps the most economical, and hence compelling [6]. In the MSSM the Higgs spectrum is richer as compared to the Higgs sector of the SM, and consists of two Higgs superfields $\left(H_{1}\right.$ and $\left.H_{2}\right)$. After spontaneous symmetry breaking the model contains two CP even Higgs bosons $\left(h^{0}, H^{0} ; M_{h^{0}}<M_{H^{0}}\right)$, one CP odd Higgs boson $\left(A^{0}\right)$, and two charged states $\left(H^{ \pm}\right)$. At the tree level the Higgs sector of MSSM is rather constrained and is described by two parameters, usually taken to be the mass of $A^{0}\left(M_{A}\right)$ and the ratio of the vacumm expectation values of the two Higgs fields, $\tan \beta \equiv\left\langle H_{2}^{0}\right\rangle /\left\langle H_{1}^{0}\right\rangle$. Discovery of more than one Higgs boson at the LHC would point towards an extension of the SM, of which supersymmetry as embodied in the minimal supersymmetric standard model is a leading candidate. It would, therefore, be important to discover an extended Higgs sector at the LHC, if it exists. However, from the point of view of supersymmetry, it is crucial to discover the supersymmetric partners of the SM states as predicted by the MSSM such as squarks, gluinos and sleptons, as well as neutralinos and charginos. In the absence of any signal for supersymmetric particles, it would, therefore, be appropriate to ask the question whether non observation of an extended Higgs sector would imply that the new physics is at a much higher scale. This question is intimately connected with the decay patterns of the Higgs bosons of the MSSM. It is possible that the Higgs bosons of the MSSM may decay into some of the lighter particles of the supersymmetric spectrum at a rapid rate. This, in particular, would include light neutralino pairs. This decay can be an important decay channel in certain regions of the MSSM parameter space. Hence we need to study the invisible decay of the second lightest Higgs boson extensively. Another aspect of this issue is that even though MSSM could be ruled out, there are appealing alternatives, namely the nonminimal or next-to-minimal supersymmetric standard model (NMSSM) whose Higgs [7-14] and neutralino [15-17] sectors are richer than the MSSM, thereby increasing the possibility of an invisibly decaying Higgs boson. Furthermore, in the NMSSM there is a possibility of a low mass pseudoscalar in the Higgs spectrum. Thus, there is a distinct possibility of the scalar Higgs boson(s) decaying invisibly into these pseudoscalars [18].

The invisible Higgs decay width has been constrained by various groups by performing fits of the signal strengths in various search channels using the latest LHC Higgs data [19, 20]. Direct searches for invisible 
decaying Higgs produced in association with a Z boson has been carried out by the ATLAS [21, 22] and CMS [23] collaborations at the LHC and they have excluded branching ratio of more than $65 \%$ and $75 \%$, respectively, with 95\% CL. The CMS collaboration [24] has also carried out a similar search for invisible branching ratio of the Higgs boson produced in the vector boson fusion process and put an upper limit of $69 \%$ on the invisible branching ratio of the lightest Higgs boson. All these searches have in turn put constraints on the MSSM parameter space. Although specific regions of the MSSM parameter space have been ruled out by these experiments, there is still a large portion of the MSSM parameter space which remains unexplored. Recently, the question of the invisible decays of the lightest Higgs boson in the context of the MSSM and the NMSSM have been discussed in detail $[18,25]$. In this paper we carry this investigation further by analyzing the decay patterns and the invisible decays of the heavier Higgs boson $\left(H^{0}\right)$ of the MSSM. For this purpose we shall identify the state observed near $126 \mathrm{GeV}$ at the CERN LHC with the lightest Higgs boson $\left(h^{0}\right)$ of the MSSM. We shall systematically study the scenarios under which such a possibility can arise and discuss different aspects of these scenarios.

The plan of the paper is as follows. In Section II we discuss the relevant features of the Higgs sector of the MSSM, and enumerate the two regions, the decoupling and the non-decoupling regions, of its parameter space. In Section III we discuss the neutralino sector and the neutralino mass matrix of the MSSM with two different kinds of grand unified theory (GUT) boundary conditions on its parameters, namely universal and nonuniversal boundary conditions and the composition of neutralinos. In Section IV we summarize the analytical expressions for the decay of second lightest Higgs boson to neutralinos in the MSSM with an appropriate choice of the parameter space, taking all the experimental contraints into account. In Section V we present our calculations and numerical results for the invisible decay of the second lightest Higgs boson and comment on how some of them may be rendered visible(quasi-invisibility). Finally in Section VI we summarize our results and conclusions.

\section{THE HIGGS SECTOR OF MINIMAL SUPERSYMMETRIC STANDARD MODEL}

The ATLAS $[1,2]$ and CMS [3, 4] experiments at the CERN LHC have independently observed a resonance, whose properties are consistent with the SM Higgs boson. The ATLAS experiment after collecting data at an integrated luminosity of $4.8 \mathrm{fb}^{-1}$ at $\sqrt{s}=7 \mathrm{TeV}$ in 2011 and $5.8 \mathrm{fb}^{-1}$ at $\sqrt{s}=8 \mathrm{TeV}$ in 2012 confirmed the evidence for the production of a neutral boson with a measured mass of $126.0 \pm 0.4$ (stat) \pm 0.4 (sys) GeV, with a significance of $5.9 \sigma$. The CMS experiment after collecting $5.1 \mathrm{fb}^{-1}$ at $7 \mathrm{TeV}$ and $5.3 \mathrm{fb}^{-1}$ at $8 \mathrm{TeV}$ reported an evidence of a neutral boson at $125.3 \pm 0.4$ (stat.) \pm 0.5 (syst.) GeV with a significance of $5.8 \sigma$. As mentioned in the Introduction, within the framework of the MSSM, we shall identify this resonance with the lightest Higgs boson of the model. We recall that the tree-level Higgs boson masses are determined by CP-odd Higgs boson mass $M_{A}$ and $\tan \beta$. Requiring the production cross section of $126 \mathrm{GeV}$ Higgs boson decaying to two photons agrees with the one observed at the CERN LHC, divides the MSSM Higgs parameter space into two distinct regions $[26]$ :

- The non-decouplng regime where $M_{A} \lesssim 130 \mathrm{GeV}$. In this region the heavier CP-even state $H^{0}$ is SMlike, and the light CP-even Higgs state $h^{0}$ and the CP-odd Higgs state $A^{0}$ are almost degenerate in mass [27] and close to $M_{Z}$, while the charged Higgs bosons are nearly degenerate with $H^{0}$ [28]. The LHC phenomenology for this sector has been discussed in the past [29-31].

- The decoupling limit where $M_{A} \gtrsim 300 \mathrm{GeV}$. In this region the light CP-even Higgs boson $h^{0}$ is SM-like, and all the other physical Higgs boson(s) are heavy and almost degenerate with $A^{0}[32]$. The decoupling properties of the MSSM Higgs sector are not special to supersymmetry. Rather, they are a generic feature of non-minimal Higgs sectors.

The non-decoupling scenario, which leads to a light SM-like Higgs, is highly constrained. Thus, the decoupling regime is a more viable scenario as far as the MSSM Higgs search results are concerned. Therefore, we shall consider only this scenario in this paper. For the non-decoupling regime of supersymmetric models, see, e.g. [25].

Having summarized the experimental results and the different scenarios, we now summarize the aspects of the MSSM Higgs sector that are relevant to our discussion. The masses $M_{h / H}$ and the mixing angle $\alpha$ of the 
neutral CP-even Higgs states are well known. These can be written as [33]

$$
\begin{aligned}
M_{h / H}^{2} & =\frac{1}{2}\left(M_{A}^{2}+M_{Z}^{2}+\Delta M_{11}^{2}+\Delta M_{22}^{2} \mp \sqrt{M_{A}^{4}+M_{Z}^{4}-2 M_{A}^{2} M_{Z}^{2} c_{4 \beta}+C}\right) \\
\tan \alpha & =\frac{2 \Delta M_{12}^{2}-\left(M_{A}^{2}+M_{Z}^{2}\right) s_{\beta}}{\Delta M_{11}^{2}-\Delta M_{22}^{2}+\left(M_{Z}^{2}-M_{A}^{2}\right) c_{2 \beta}+\sqrt{M_{A}^{4}+M_{Z}^{4}-2 M_{A}^{2} M_{Z}^{2} c_{4 \beta}+C}},
\end{aligned}
$$

where

$$
C=4 \Delta M_{12}^{4}+\left(\Delta M_{11}^{2}-\Delta M_{22}^{2}\right)^{2}-2\left(M_{A}^{2}-M_{Z}^{2}\right)\left(\Delta M_{11}^{2}-\Delta M_{22}^{2}\right) c_{2 \beta}-4\left(M_{A}^{2}+M_{Z}^{2}\right) \Delta M_{12}^{2} s_{2 \beta},
$$

and $c_{4 \beta} \equiv \cos 4 \beta, c_{2 \beta} \equiv \cos 2 \beta, c_{\beta} \equiv \cos \beta$, and $s_{\beta} \equiv \sin \beta$. Furthermore, $\Delta M_{i j}, i, j=1,2$ quantify the radiative corrections to the CP-even Higgs boson mass matrix.

The dominant radiative corrections arising from the top-stop sector are contained in $\Delta M_{22}$. Identifying the resonance discovered at $126 \mathrm{GeV}$ with $h^{0}$, one can, then, write the dominant radiative correction to the Higgs boson mass matrix in terms of the mass $M_{h}$ of $h^{0}$ as

$$
\Delta M_{22}^{2}=\frac{M_{h}^{2}\left(M_{A}^{2}+M_{Z}^{2}-M_{h}^{2}\right)-M_{A}^{2} M_{Z}^{2} c_{2 \beta}^{2}}{M_{Z}^{2} c_{\beta}^{2}+M_{A}^{2} s_{\beta}^{2}-M_{h}^{2}},
$$

where we have used the approximation $\Delta M_{22} \gg \Delta M_{11}, \Delta M_{12}$, and where $M_{A}$ is the mass of the pseudoscalar Higgs boson $A^{0}$. We note that there is another solution for $\Delta M_{22}^{2}$, which is unphysical [34], and is, therefore, not relevant.

With this approximation we can write the mass of $H^{0}$ and the mixing angle $\alpha$ in terms of $M_{A}$ and tan $\beta$ as

$$
\begin{aligned}
M_{H}^{2} & =\frac{\left(M_{A}^{2}+M_{Z}^{2}-M_{h}^{2}\right)\left(M_{Z}^{2} c_{\beta}^{2}+M_{A}^{2} s_{\beta}^{2}\right)-M_{A}^{2} M_{Z}^{2} c_{2 \beta}^{2}}{M_{Z}^{2} c_{\beta}^{2}+M_{A}^{2} s_{\beta}^{2}-M_{h}^{2}}, \\
\alpha & =-\tan ^{-1}\left(\frac{\left(M_{Z}^{2}+M_{A}^{2}\right) c_{\beta} s_{\beta}}{M_{Z}^{2} c_{\beta}^{2}+M_{A}^{2} s_{\beta}^{2}-M_{h}^{2}}\right) .
\end{aligned}
$$

Thus, in principle one can calculate the mass of $H^{0}$. However, in actual practice the mass of $H^{0}$ will depend on other parameters of the model, which include the supersymmetry conserving Higgs bilinear parameter $\mu$, and the supersymmetry breaking scale $M_{S}$. In order to obtain a handle on the behavior of solutions of interest to us, we perform a numerical scan using the package CalcHEP [35]. For our study we use a set of inputs which are consistent with the known experimental constraints, and also which have the possibility of leading to spectra that may be visible in the near future. Thus, we are guided by the principle of search for SUSY in upcoming experiments and choices of parameters that continue to make low energy SUSY a viable option to address the naturalness and hierarchy problems of the SM. We have calculated the dependence of $M_{H}$ on $\mu$ for different values of $\tan \beta$ and the supersymmetry breaking scale $M_{S}$. In Figs. 1 and 2 we show the dependence of $M_{H}$ on $\mu$ for values of $M_{A}=300 \mathrm{GeV}$ and $M_{A}=500 \mathrm{GeV}$. From these two figures we conclude that $M_{H}$ does not vary significantly as a function of $\mu$. For fixed values of $\tan \beta$ it has a weak dependence on $M_{S}$. However, $M_{H}$ has a significant dependence on $M_{A}$ and $\tan \beta$, and can be described fully in terms of these two parameters when we use the fact that $M_{h}$ is in the range 123-129 GeV. This feature had been pointed out earlier in Refs. [36-38]. Our results agree with these authors. In the present work these results have been obtained numerically by using as input the parameters $M_{S}, \tan \beta$ and $\mu$ as detailed above, and adjusting the scalar trilinear coupling $A_{t}$ so as to have $M_{h}$ in the range $123-129 \mathrm{GeV}$. We have checked that the results of our calculations are in good agreement with those of [33].

\section{THE NEUTRALINO SECTOR OF THE MSSM WITH GUT BOUNDARY CONDITIONS}

In order to study the invisible decays of $H^{0}$ to neutralinos, we consider the neutralino sector of the MSSM in some detail. For this purpose we consider the neutralino mass matrix of the model and the implications of the GUT boundary conditions on the neutralino spectrum. This analysis will lead to a general understanding of the nature of mixing between the gaugino and Higgsino states, as well as those of the physical neutralino states after electroweak symmetry breaking.

We recall that the neutralinos are an admixture of the fermionic partners of the two Higgs doublets, $H_{1}$ and $H_{2}$, and the fermionic partners of the neutral gauge bosons. When the electroweak symmetry is broken, the 


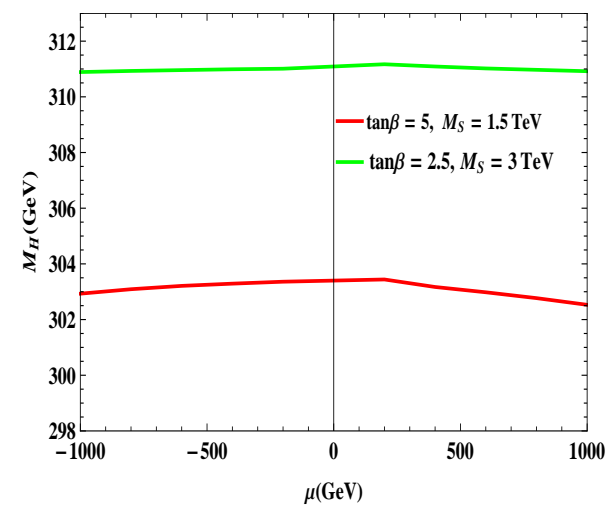

FIG. 1: $M_{H}$ as a function of $\mu$ for $M_{A}=300$

$\mathrm{GeV}$ for two different values of $\tan \beta$ and the supersymmetry breaking scale $M_{S}$.

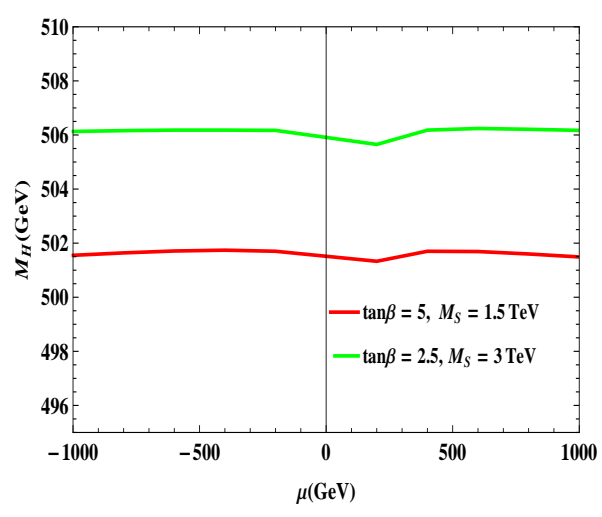

FIG. 2: $M_{H}$ as a function of $\mu$ for $M_{A}=500$

$\mathrm{GeV}$ for two different values of $\tan \beta$ and the supersymmetry breaking scale $M_{S}$.

physical mass eigenstates are obtained from the diagonalization of the neutralino mass matrix [39-41]

$$
M_{\mathrm{MSSM}}=\left(\begin{array}{cccc}
M_{1} & 0 & -m_{Z} \sin \theta_{W} \cos \beta & m_{Z} \sin \theta_{W} \sin \beta \\
0 & M_{2} & m_{Z} \cos \theta_{W} \cos \beta & -m_{Z} \cos \theta_{W} \sin \beta \\
-m_{Z} \sin \theta_{W} \cos \beta & m_{Z} \cos \theta_{W} \cos \beta & 0 & -\mu \\
m_{Z} \sin \theta_{W} \sin \beta & -m_{Z} \cos \theta_{W} \sin \beta & -\mu & 0
\end{array}\right),
$$

where $M_{1}$ and $M_{2}$ are the $U(1)_{Y}$ and the $S U(2)_{L}$ soft supersymmetry breaking gaugino mass parameters, $\mu$ is the Higgs(ino) mass parameter, $M_{Z}$ is the $Z$ boson mass, $\theta_{W}$ is the weak mixing angle and $\tan \beta \equiv v_{2} / v_{1}$ is the ratio of the vacuum expectation values of the neutral components of the two Higgs doublet fields $H_{1}$ and $H_{2}$. The gaugino mass parameters $M_{1}$ and $M_{2}$ may have some relation between them, or they can be completely arbitrary. If we assume that the MSSM is embedded in a grand unified theory, then the boundary conditions at the GUT scale imply a definite relation between the gaugino masses, which would imply a relation between them at the weak scale following the renormalization group evolution. Here we will consider two types of boundary conditions on $M_{i}$ that follow from embedding of MSSM in a grand unified theory, namely the universal boundary conditions and the nonuniversal boundary conditions.

\section{A. Universal boundary condition}

In the MSSM, with universal gaugino masses at the grand unified scale, usually referred to as mSUGRA, the soft supersymmetry breaking gaugino mass parameters $M_{i}$ and the corresponding gauge couplings $\alpha_{i}(i=1,2,3)$ satisfy the boundary condition

$$
\begin{aligned}
M_{1} & =M_{2}=M_{3}=m_{1 / 2}, \\
\alpha_{1} & =\alpha_{2}=\alpha_{3}=\alpha_{G},
\end{aligned}
$$

at the grand unified scale $M_{G}$. Using the one-loop renormalization group equations [42] for the gaugino masses and the gauge couplings leads to the ratio

$$
M_{1}: M_{2}: M_{3} \simeq 1: 2: 7.1 \text {, }
$$

for the soft gaugino masses at the electroweak scale $M_{Z}$. In the following, for definiteness, we shall consider the value of $\tan \beta=10$. If one is interested in Higgs boson decaying invisibly into light neutralinos, it is appropriate to consider a light or massless eigenstate of the mass matrix (III.1). It has been shown earlier [18, 25] that with the gaugino mass parameters satisfying the universal boundary conditions at the GUT scale, it is not possible to satisfy the masslessness condition for the lightest neutralino following from the determinant of the mass matrix (III.1) and the experimental constraints as implied by the LEP experiments [43]. Thus, a massless neutralino is ruled out with universal boundary conditions (III.3) at the GUT scale. 


\begin{tabular}{||c|ccc|ccc||}
\hline$S U(5)$ & $M_{1}^{G}$ & $M_{2}^{G}$ & $M_{3}^{G}$ & $M_{1}^{E W}$ & $M_{2}^{E W}$ & $M_{3}^{E W}$ \\
\hline $\mathbf{1}$ & 1 & 1 & 1 & 1 & 2 & 7.1 \\
$\mathbf{2 4}$ & 1 & 3 & -2 & 1 & 6 & -14.3 \\
$\mathbf{7 5}$ & 1 & $-\frac{3}{5}$ & $-\frac{1}{5}$ & 1 & -1.18 & -1.41 \\
$\mathbf{2 0 0}$ & 1 & $\frac{1}{5}$ & $\frac{1}{10}$ & 1 & 0.4 & 0.71 \\
\hline
\end{tabular}

TABLE I: Ratios of the gaugino masses at the GUT scale in the normalization $M_{1}(G U T)=1$, and at the electroweak scale in the normalization $M_{1}(E W)=1$ for $F$-terms in different representations of $S U(5)$. These results are obtained by using 1-loop renormalization group equations.

\section{B. Non-universal boundary condition}

Universal soft supersymmetry breaking gaugino masses are not the only possibility in a grand unified theory. In fact, non universal boundary conditions for the soft gaugino masses can arise quite naturally in $S U(5), S O(10)$ and $E_{6}$ supersymmetric grand unified theories.

In grand unified supersymmetric models, gaugino masses are generated by a non-singlet chiral superfield $\Phi^{n}$ that appears linearly in the gauge kinetic function $f(\Phi)$, which is an analytic function of the chiral superfields $\Phi$ in the theory[44]. The gaugino masses are generated from the coupling of the field strength superfield $W^{a}$ with $f(\Phi)$, when the auxiliary part $F_{\Phi}$ of a chiral superfield $\Phi$ in $f(\Phi)$ gets a VEV. When $F_{\Phi}$ gets a VEV $\left\langle F_{\Phi}\right\rangle$, we obtain the Lagrangian containing the gaugino masses

$$
\mathcal{L}_{g . k .} \supset \frac{\left\langle F_{\Phi}\right\rangle_{a b}}{M_{P}} \lambda^{a} \lambda^{b}+\text { h.c. },
$$

where $\lambda^{a, b}$ are gaugino fields. Here, we denote by $\lambda^{1}, \lambda^{2}$ and $\lambda^{3}$ the $U(1)_{Y}, S U(2)_{L}$ and $S U(3)_{C}$ gaugino fields, respectively. Since the gauginos belong to the adjoint representation of the gauge group, $\Phi$ and $F_{\Phi}$ can belong to any of the representations appearing in the symmetric product of the two adjoint representations of the unified gauge group.

For example, in the case where the SM gauge group is embedded in the grand unified gauge group $S U(5)$ the symmetric product of the two adjoint ( $\mathbf{2 4}$ dimensional) representations of $S U(5)$ leads to

$$
(\mathbf{2 4} \otimes \mathbf{2 4})_{\text {Symm }}=\mathbf{1} \oplus \mathbf{2 4} \oplus \mathbf{7 5} \oplus \mathbf{2 0 0} .
$$

In Table I we have summarized the ratios of gaugino masses which result when $F_{\Phi}$ belongs to different representations of $S U(5)$ in the decomposition (III.6).

Similarly, nonuniversal gaugino masses can arise from the embedding of MSSM in a grand unified theory based on $S O(10)$ and $E_{6}$. For these gauge groups we have the decomposition

$S O(10)$ :

$$
(45 \otimes \mathbf{4 5})_{S y m m}=1 \oplus \mathbf{5 4} \oplus \mathbf{2 1 0} \oplus \mathbf{7 7 0} ;
$$

$E_{6}:$

$$
(\mathbf{7 8} \otimes \mathbf{7 8})_{S y m m}=\mathbf{1} \oplus \mathbf{6 5 0} \oplus \mathbf{2 4 3 0} .
$$

The analogs of Table I for the gauge groups $\mathrm{SO}(10)$ and $E_{6}$ are given in Appendix A of [18], where a detailed discussion has been presented on the phenomenological consequences of different choices of the grand unified gauge group. We note that in the present work we are not necessarily looking at very light neutralinos as the second lightest Higgs boson can be heavy, and can decay into neutralinos that are massive.

\section{Composition of Neutralinos}

The composition of the lightest neutralino $\tilde{\chi}_{1}^{0}$ in terms of the gauginos and Higgsinos can be written as [45, 46]

$$
\tilde{\chi}_{1}^{0}=Z_{11} \tilde{B}+Z_{12} \tilde{W}^{3}+Z_{13} \tilde{H}_{1}^{0}+Z_{14} \tilde{H}_{2}^{0}
$$




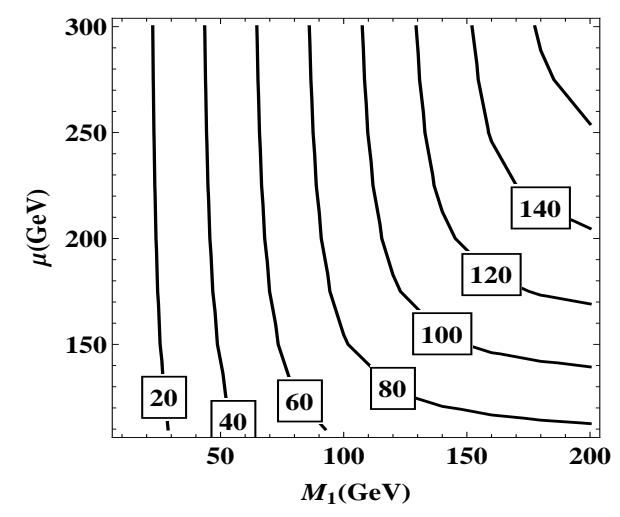

FIG. 3: The contours of constant lightest neutralino mass $m_{\tilde{\chi}_{1}^{0}}$ in MSSM with arbitrary gaugino masses at the GUT scale in the $\mu-M_{1}$ plane. Here the value of the parameter $M_{2}=200$ $\mathrm{GeV}$.

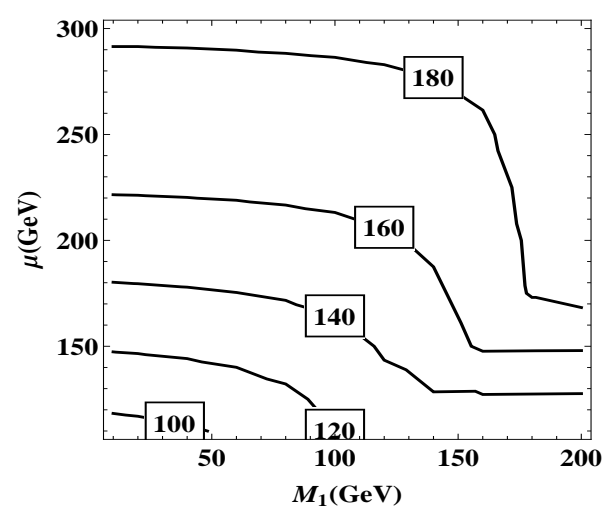

FIG. 4: The contours of constant second lightest neutralino mass $m_{\tilde{\chi}_{2}^{0}}$ in MSSM with arbitrary gaugino masses at the GUT scale in the $\mu-M_{1}$ plane. Here the value of $M_{2}=200 \mathrm{GeV}$.

where

$$
Z_{1 i}=\left(1,-\frac{\cot \theta_{W}\left(M_{1}-m_{\chi_{1}^{0}}\right)}{M_{2}-m_{\chi_{1}^{0}}}, \frac{\left(M_{1}-m_{\chi_{1}^{0}}\right)\left(\mu \sin \beta+m_{\chi_{1}^{0}} \cos \beta\right)}{m_{Z} \sin \theta_{W}\left(m_{\chi_{1}^{0}}+\mu \sin 2 \beta\right)},-\frac{\left(M_{1}-m_{\chi_{1}^{0}}\right)\left(\mu \cos \beta+m_{\chi_{1}^{0}} \sin \beta\right)}{m_{Z} \sin \theta_{W}\left(m_{\chi_{1}^{0}}+\mu \sin 2 \beta\right)}\right) .
$$

The composition of the second lightest neutralino can be written as

$$
\tilde{\chi}_{2}^{0}=Z_{21} \tilde{B}+Z_{22} \tilde{W}^{3}+Z_{23} \tilde{H}_{1}^{0}+Z_{24} \tilde{H}_{2}^{0}
$$

where

$$
Z_{2 i}=\left(1,-\frac{\cot \theta_{W}\left(M_{1}-m_{\chi_{2}^{0}}\right)}{M_{2}-m_{\chi_{2}^{0}}}, \frac{\left(M_{1}-m_{\chi_{2}^{0}}\right)\left(\mu \sin \beta+m_{\chi_{2}^{0}} \cos \beta\right)}{m_{Z} \sin \theta_{W}\left(m_{\chi_{2}^{0}}+\mu \sin 2 \beta\right)},-\frac{\left(M_{1}-m_{\chi_{2}^{0}}\right)\left(\mu \cos \beta+m_{\chi_{2}^{0}} \sin \beta\right)}{m_{Z} \sin \theta_{W}\left(m_{\chi_{2}^{0}}+\mu \sin 2 \beta\right)}\right) .
$$

The possibility of $H^{0}$ decaying into neutralinos depends on the mass of the neutralinos into which it can decay. Our objective is to study the invisible decay of the second lightest Higgs boson into neutralinos. For this purpose, we carry out a study of the neutralino mass as a function of $M_{1}$ and $\mu$, as these parameters, apart from $M_{2}$, control the neutralino mass matrix, and hence the neutralino mass eigenvalues. We have considered the constant mass curves for both the lightest and the second lightest neutralinos. Furthermore, we have considered each of the scenarios, namely the case of arbitrary gaugino masses, universal, and non-universal gaugino masses, respectively at the grand unified scale in Figs. 3, 4, 5, 6, 7 and 8 .

For arbitrary supersymmetry breaking gaugino masses at the grand unified scale, we shall choose the parameter $M_{2}=200 \mathrm{GeV}$, a choice we shall discuss in the following. It is obvious that choosing universal or nonuniversal boundary conditions at the grand unified scale is a rather restrictive condition.

In Figs. 3 and 4 we plot the contours of constant lightest and the second lightest neutralino masses in the $\mu-M_{1}$ plane for arbitrary gaugino masses at the GUT scale. It is clear that $m_{\tilde{\chi}_{1}^{0}}$ increases with $M_{1}$ but does not change significantly with $\mu$. On the other hand the second lightest neutralino mass $m_{\tilde{\chi}_{2}^{0}}$ does not change appreciably with $M_{1}$ but increases with $\mu$. This is primarily because $\tilde{\chi}_{1}^{0}$ is bino-like, and $\tilde{\chi}_{2}^{0}$ is a Higgsinolike state. For low values of $M_{1}$, the mass of $\tilde{\chi}_{1}^{0}$ is almost equal to $M_{1}$, and it is bino-like for all values of $\mu$. Furthermore, when $M_{1}>\mu, \tilde{\chi}_{1}^{0}$ is Higgsino-like. At large $\mu$, and when $M_{1}>M_{2}(200 \mathrm{GeV}), \tilde{\chi}_{1}^{0}$ will be wino-like. We see from Fig. 3 that with arbitrary gaugino masses and with $M_{1}$ larger than $150 \mathrm{GeV}$ and $\mu$ larger than $250 \mathrm{GeV}$ the mass of $\tilde{\chi}_{1}^{0}$ approaches $150 \mathrm{GeV}$ and, thus, beyond these values a $300 \mathrm{GeV}$ Higgs cannot decay into two lightest neutralinos.

For low values of $M_{1}$ and with $\mu<M_{2}(200 \mathrm{GeV}), \tilde{\chi}_{2}^{0}$ is Higgsino-like. Furthermore, for values of $\mu>$ $M_{2}(200 \mathrm{GeV}), \tilde{\chi}_{2}^{0}$ is wino-like for all values of $M_{1}$. For values of $\mu<M_{1}, \tilde{\chi}_{2}^{0}$ is Higgsino-like. In Fig. 4 we see that for $M_{1} \geq 150 \mathrm{GeV}$ and $\mu \geq 200 \mathrm{GeV}$, the mass of $\tilde{\chi}_{2}^{0}$ approaches $150 \mathrm{GeV}$, and hence for values larger than these a $300 \mathrm{GeV}$ second lightest Higgs cannot decay into two second lightest neutralinos. Thus, only when 


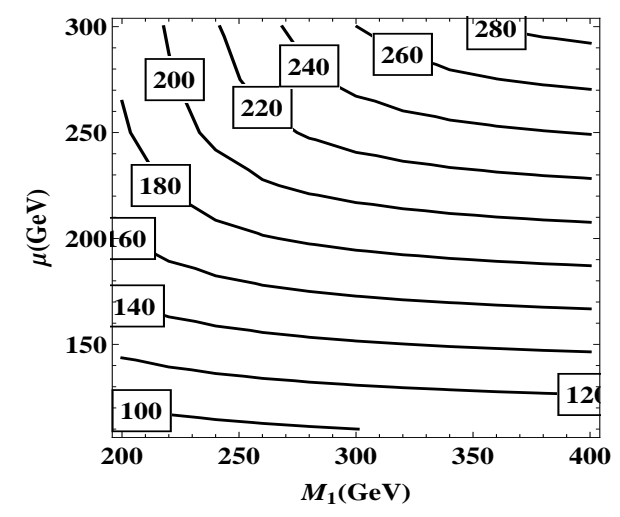

FIG. 5: The contours of constant lightest neutralino mass $m_{\tilde{\chi}_{1}^{0}}$ in the $\mu-M_{1}$ plane in MSSM with universal soft gaugino masses at the GUT scale.

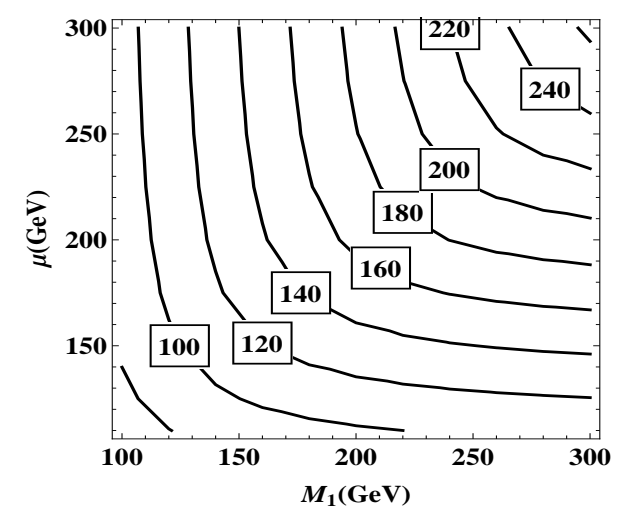

FIG. 7: The contours of constant lightest neutralino mass $m_{\tilde{\chi}_{1}^{0}}$ in the $\mu-M_{1}$ plane with

MSSM embedded in $\mathrm{SO}(10)$, and with non-universal soft gaugino masses at the GUT scale.

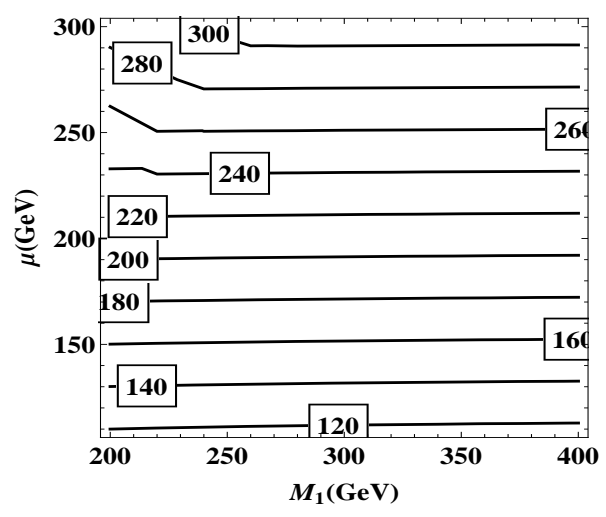

FIG. 6: The contours of constant second lightest neutralino mass $m_{\tilde{\chi}_{2}^{0}}$ in the $\mu-M_{1}$ plane in MSSM with universal soft gaugino masses at the GUT scale.

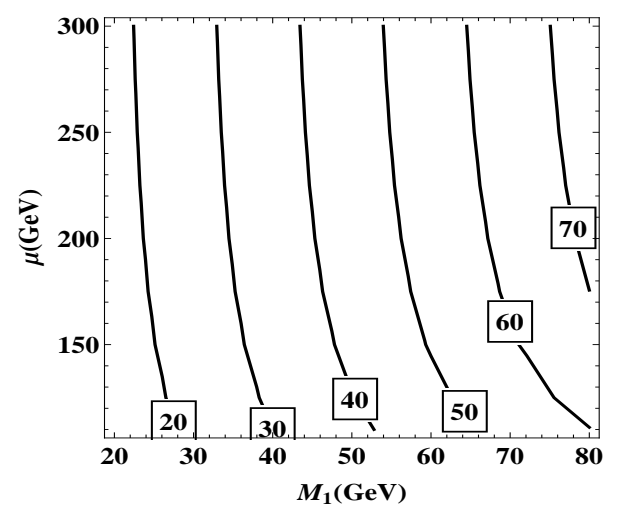

FIG. 8: The contours of constant second lightest neutralino mass $m_{\tilde{\chi}_{1}^{0}}$ in the $\mu-M_{1}$ plane with MSSM embedded in $E_{6}$, and with non-universal soft gaugino masses at the GUT scale.

$M_{1}$ is large and $\mu$ is relatively small, or if $\mu$ is large and $M_{1}$ is relatively small, $H^{0}$ can decay invisibly into neutralinos.

It is instructive to consider here the case of universal boundary conditions on the gaugino masses at the grand unified scale. Considering the values of $M_{1}$ in the range $200-400 \mathrm{GeV}$, which implies values of $M_{2}$ in the range of $400-800 \mathrm{GeV}$, for low values of $\mu, \tilde{\chi}_{1}^{0}$ is Higgsino-like, and for large values of $\mu$, where $\mu>M_{1}, \mu, m_{\tilde{\chi}_{1}^{0}}$ is bino-like. Similarly, for values of $M_{2}>M_{1}, \mu m_{\tilde{\chi}_{2}^{0}}$ is Higgsino-like. In Fig. 5 and Fig. 6 we plot the contours of constant neutralino masses in the $\mu-M_{1}$ plane for the case of universal boundary conditions on the soft gaugino masses at the grand unified scale. Here $m_{\tilde{\chi}_{1}^{0}}$ and $m_{\tilde{\chi}_{2}^{0}}$ do not change significantly as a function of $M_{1}$. However, both the masses are an increasing function of $\mu$. From Fig. 5 we see that with $\mu$ larger than $175 \mathrm{GeV}$, $m_{\tilde{\chi}_{1}^{0}}$ approaches $150 \mathrm{GeV}$, and, thus, a $300 \mathrm{GeV}$ Higgs cannot decay into two lightest neutralinos for the case of universal boundary conditions. For values of $\mu$ larger than $275 \mathrm{GeV} m_{\tilde{\chi}_{1}^{0}}$ approaches $250 \mathrm{GeV}$ for $M_{1} \geq 300$ $\mathrm{GeV}$. Hence, for these values of $\mu$, a $500 \mathrm{GeV}$ Higgs cannot decay into lightest neutralinos.. However, for low $M_{1}$ larger values of $\mu$ will be allowed. In Fig. 6 we can see $m_{\tilde{\chi}_{2}^{0}}$ dominantly depends on $\mu$. For $\mu$ larger than $150 \mathrm{GeV}, m_{\tilde{\chi}_{2}^{0}}$ crosses the $150 \mathrm{GeV}$ limit and the decay of the $300 \mathrm{GeV}$ second lightest Higgs into two second lightest neutralinos will be forbidden for these values of $\mu$ for any value of $M_{1}$. Similarly, for a $500 \mathrm{GeV}$ Higgs boson, $\mu=250 \mathrm{GeV}$ is the limit because for higher values of $\mu, m_{\tilde{\chi}_{2}^{0}}$ approaches a value of $250 \mathrm{GeV}$.

In Fig. 7 and 8 we discuss two typical examples of nonuniversal boundary conditions on the gaugino masses at the GUT scale. The first is the case of SO(10) GUT with the condition that the three gaugino masses are in the ratio $M_{1}: M_{2}: M_{3}=1: 6:-14.3$, and the second one is with $E_{6}$ GUT with the boundary condition in which the three gaugino masses are in the ratio $M_{1}: M_{2}: M_{3}=1: 50.2: 70.9$. The case of $S O(10)$ is 
akin to that universal boundary condtion discussed above because the range of $M_{2}$ values considered is greater than the range of $M_{1}$ and $\mu$. The range of values of $M_{1}$ considered here is $100-300 \mathrm{GeV}$. For $M_{1}<\mu$, $\tilde{\chi}_{1}^{0}$ is bino dominated, and for $M_{1}>\mu, \tilde{\chi}_{1}^{0}$ is Higgsino dominated. On the other hand, the particular choice of $E_{6}$ representation is very interesting because this is the only GUT representation in which the lightest CP-even Higgs $h^{0}$ can decay into two lightest neutralinos. With $E_{6}$ boundary condition we can see that the mass of the lightest neutralinos does not depend on $\mu$, whereas in the case of $\mathrm{SO}(10)$ the mass depends on both $M_{1}$ and $\mu$. For this $E_{6}$ representation the range of $M_{1}$ values considered here is $20-80 \mathrm{GeV}$, and values of $M_{1}<\mu, M_{2}$ for the entire parameter space. Hence in this case $\tilde{\chi}_{1}^{0}$ is always bino-like.

We recall here that there are additional constraints coming from the LEP analysis of the $Z^{0}$ decay in invisible modes, on the $\mu-M_{1}$ parameter space. The invisible decay of the lightest Higgs boson to the lightest neutralinos, if kinematically allowed, is mainly constrained by the $Z$ invisible decay rate. This invisible decay width has been measured very precisely by the LEP experiments [43] with

$$
\Gamma\left(Z^{0} \rightarrow \tilde{\chi}_{1}^{0} \tilde{\chi}_{1}^{0}\right)<3 \mathrm{MeV}
$$

The $Z$ width to a pair of lightest neutralinos can be written as [47]

$$
\Gamma\left(Z^{0} \rightarrow \tilde{\chi}_{1}^{0} \tilde{\chi}_{1}^{0}\right)=\frac{G_{F} m_{Z}^{3}}{6 \sqrt{2} \pi}\left(Z_{13}^{2}-Z_{14}^{2}\right)\left(1-\frac{4 m_{\tilde{\chi}_{1}^{0}}^{2}}{m_{Z^{0}}^{2}}\right)^{3 / 2}
$$

For our analysis we have taken $\tan \beta=10$ [28]. The trilinear soft supersymmetry breaking coupling $A_{t}$ has been adjusted in order to obtain a lightest Higgs boson mass of $\approx 126 \mathrm{GeV}$. The gluino mass is taken to be $1400 \mathrm{GeV}$ [48], and the squarks are assumed to have a mass above $1 \mathrm{TeV}$ [49], thereby respecting the current experimental bounds.

In our calculations we have imposed the constraint of the lightest chargino mass bound of $m_{\tilde{\chi}^{+}}>94 \mathrm{GeV}$ following from the LEP experiments as well as the bound from invisible $Z^{0}$ decay width coming from $Z^{0}$ decay into neutralinos [50].

Having discussed in some detail the correlations among the parameters of the neutralino sector, we now turn to the implications of this analysis for the possible invisible decays of $H^{0}$ for a choice of parameters which are consistent with the constraints discussed in this section.

\section{DECAY OF THE SECOND LIGHTEST HIGGS BOSON TO NEUTRALINOS IN THE MINIMAL SUPERSYMMETRIC STANDARD MODEL.}

We now address the main issue of this paper, namely the invisible decays of the heavier CP-even Higgs boson $H^{0}$ into neutralinos in the MSSM $[51,52]$. We consider these decays in the decoupling regime of the MSSM, where the Higgs boson $H^{0}$ is relatively heavy. In the decoupling regime, the heavy $H^{0}$ can decay into neutralinos which are not necessarily light.

For this purpose we catalog the decay widths of the heavier CP-even Higgs boson into a pair of lighter neutralinos in the MSSM. These can be written as

$$
\Gamma\left(H^{0} \rightarrow \tilde{\chi}_{1}^{0} \tilde{\chi}_{1}^{0}\right)=\frac{G_{F} M_{W}^{2} M_{H}}{2 \sqrt{2} \pi}\left(1-4 m_{\tilde{\chi}_{1}^{0}}^{2} / M_{H}^{2}\right)^{3 / 2}\left[\left(Z_{12}-\tan \theta_{W} Z_{11}\right)\left(Z_{13} \cos \alpha-Z_{14} \sin \alpha\right)\right]^{2},
$$

where $Z_{i j}$ are the elements of the matrix $Z$ which diagonalizes the neutralino mass matrix (III.1). In the decoupling limit, when the mass of the pseudoscalar $A^{0}$ is large compared to the mass of the $Z$ boson $M_{Z}$, the Higgs mixing angle $\alpha \rightarrow \beta-\pi / 2$, so that the decay width (IV.1) can be written as

$$
\Gamma\left(H^{0} \rightarrow \tilde{\chi}_{1}^{0} \tilde{\chi}_{1}^{0}\right)=\frac{G_{F} M_{W}^{2} M_{H}}{2 \sqrt{2} \pi}\left(1-4 m_{\tilde{\chi}_{1}^{0}}^{2} / M_{H}^{2}\right)^{3 / 2}\left[\left(Z_{12}-\tan \theta_{W} Z_{11}\right)\left(Z_{13} \sin \beta+Z_{14} \cos \beta\right)\right]^{2}
$$

Similarly, the decay width of $H^{0}$ into a pair of second lightest neutralinos can be written as

$$
\Gamma\left(H^{0} \rightarrow \tilde{\chi}_{2}^{0} \tilde{\chi}_{2}^{0}\right)=\frac{G_{F} M_{W}^{2} M_{H}}{2 \sqrt{2} \pi}\left(1-4 m_{\tilde{\chi}_{2}^{0}}^{2} / M_{H}^{2}\right)^{3 / 2}\left[\left(Z_{22}-\tan \theta_{W} Z_{21}\right)\left(Z_{23} \cos \alpha-Z_{24} \sin \alpha\right)\right]^{2}
$$


which in the decoupling regime becomes

$$
\Gamma\left(H^{0} \rightarrow \tilde{\chi}_{2}^{0} \tilde{\chi}_{2}^{0}\right)=\frac{G_{F} M_{W}^{2} M_{H}}{2 \sqrt{2} \pi}\left(1-4 m_{\tilde{\chi}_{2}^{0}}^{2} / M_{H}^{2}\right)^{3 / 2}\left[\left(Z_{22}-\tan \theta_{W} Z_{21}\right)\left(Z_{23} \sin \beta+Z_{24} \cos \beta\right)\right]^{2} .
$$

On the other hand the decay width for the process $H^{0} \rightarrow \chi_{1}^{0} \chi_{2}^{0}$ can be written as

$$
\begin{aligned}
\Gamma\left(H^{0} \rightarrow \tilde{\chi}_{1}^{0} \tilde{\chi}_{2}^{0}\right)= & \frac{G_{F} M_{W}^{2} M_{H}}{\sqrt{2} \pi} F_{121}^{2}\left[1+\left(m_{\tilde{\chi}_{1}^{0}}^{2} / M_{H}^{2}-m_{\tilde{\chi}_{2}^{0}}^{2} / M_{H}^{2}\right)^{2}-2\left(m_{\tilde{\chi}_{1}^{0}}^{2} / M_{H}^{2}+m_{\tilde{\chi}_{2}^{0}}^{2} / M_{H}^{2}\right)\right]^{1 / 2} \\
& \times\left[1-m_{\tilde{\chi}_{1}^{0}}^{2} / M_{H}^{2}-m_{\tilde{\chi}_{2}^{0}}^{2} / M_{H}^{2}-2\left(\epsilon_{1} \epsilon_{2} / M_{H}^{2}\right) m_{\tilde{\chi}_{1}^{0}} m_{\tilde{\chi}_{2}^{0}}\right],
\end{aligned}
$$

where

$$
F_{121}=\frac{1}{2}\left(Z_{22}-\tan \theta_{W} Z_{21}\right)\left(Z_{13} \cos \alpha-Z_{14} \sin \alpha\right)+\frac{1}{2}\left(Z_{12}-\tan \theta_{W} Z_{11}\right)\left(Z_{23} \cos \alpha-Z_{24} \sin \alpha\right),
$$

which in the decoupling regime reduces to

$$
F_{121}=\frac{1}{2}\left(Z_{22}-\tan \theta_{W} Z_{21}\right)\left(Z_{13} \sin \beta+Z_{14} \cos \beta\right)+\frac{1}{2}\left(Z_{12}-\tan \theta_{W} Z_{11}\right)\left(Z_{23} \sin \beta+Z_{24} \cos \beta\right),
$$

and where the constants $\epsilon_{i} \mathrm{~s}(\mathrm{i}=1,2,3,4)$ stand for the sign of the neutralino mass. When the neutralino mass matrix is diagonalized, we allow the sign of the ith eigenvalue to be either positive or negative.

In our previous work [18], we had considered the possibility of the lightest Higgs boson decaying into lightest neutralinos in the MSSM and the NMSSM. As the mass of the lightest Higgs boson is fixed to be $126 \mathrm{GeV}$, we had considered the case of the lightest neutralino with mass $m_{\tilde{\chi}_{1}^{0}}<m_{h^{0} / 2}$. We had concluded that such a constraint on the neutralino mass is not satisfied with either universal or nonuniversal boundary conditions on the soft gaugino masses at the GUT scale. The only exception to this conclusion was a nonuniversal scenario with a very large dimensional representation $(\mathbf{2 4 3 0})$ of the gauge group $E_{6}$. However, this is not an appealing possibility. The constraint on the light neutralino masses was obeyed in the MSSM only with arbitrary gaugino masses at the GUT scale. Since in the present paper we are considering the invisible decay of the heavier Higgs boson $H^{0}$, the lightest neutralinos need not be massless or very light. Thus, in the following we shall consider the case of massive neutralinos, which can arise in all the three cases, i.e. universal, nonuniversal and arbitrary soft gaugino masses at the GUT scale.

\section{RESULTS FOR THE INVISIBLE DECAY OF THE HEAVIER HIGGS BOSON}

Having summarized the results for the decay widths for the invisible decay of the heavier Higgs boson in the previous section, we now evaluate these decay widths using the parameter space of the MSSM allowed by the present experimental constraints. For this purpose, we shall use the boundary conditions as implied by the embedding of MSSM in a grand unified theory, as well as for arbitrary soft gaugino masses at the GUT scale. As discussed in the last section, the final states in the decay process that are of interest to us are $\chi_{i}^{0} \chi_{j}^{0}, i=1,2$.

We first summarize the parameter space used in our analysis. The trilinear soft supersymmetry breaking parameter $A_{t}$ pertaining to the stop is adjusted to obtain the lightest Higgs boson mass in the range 123-127 $\mathrm{GeV}$, although it does not affect the neutralino sector. To get the lightest Higgs mass in this range we use the "maximal mixing" scenario [53], wherein

$$
A_{t}-\mu \cot \beta=\sqrt{6} M_{S}
$$

where $M_{S}$ is the soft supersymmetry-breaking scale, which we take to be $1.5 \mathrm{TeV}$. The gluino mass is taken to be $1.4 \mathrm{TeV}$, and squarks are assumed to have masses above $1 \mathrm{TeV}$. The Slepton masses are assumed to be greater than $500 \mathrm{GeV}$, thereby respecting all current experimental bounds [54]. For the case of arbitrary boundary condition on the gaugino mass parameters, we have taken $M_{2}=200 \mathrm{GeV}$. Furthermore, we have also imposed the constraint of the lightest chargino mass $m_{\tilde{\chi}^{+}}>94 \mathrm{GeV}$ as implied by the LEP experiments, as well as the bound on the invisible $Z^{0}$ width into neutralinos. We note that decreasing the value of $M_{2}$ increases the chargino mass bound on $\mu$, thereby eliminating the parameter space with large invisible branching ratio for the lightest Higgs boson [50].

In addition, we have also imposed the constraints resulting from (g-2) of the muon, as well as flavor constraints following from $b \rightarrow s \gamma$ and $B_{s} \rightarrow \mu^{+} \mu^{-}$. We have implemented these constraints using CalcHEP. The input parameters so obtained are summarized in Table II $[55,56]$ 


\begin{tabular}{|c|c|c|c|}
\hline $\tan \beta=10$ & $M_{S}=1.5 \mathrm{TeV}$ & $M_{A}=300,500 \mathrm{GeV}$ & $M_{2}=200 \mathrm{GeV}$ \\
\hline$M_{3}=1402 \mathrm{GeV}$ & $A_{t}=3600 \mathrm{GeV}$ & $A_{b}=3600 \mathrm{GeV}$ & $A_{\tau}=1000 \mathrm{GeV}$ \\
\hline
\end{tabular}

TABLE II: Input parameters for the MSSM consistent with all the experimental constraints.

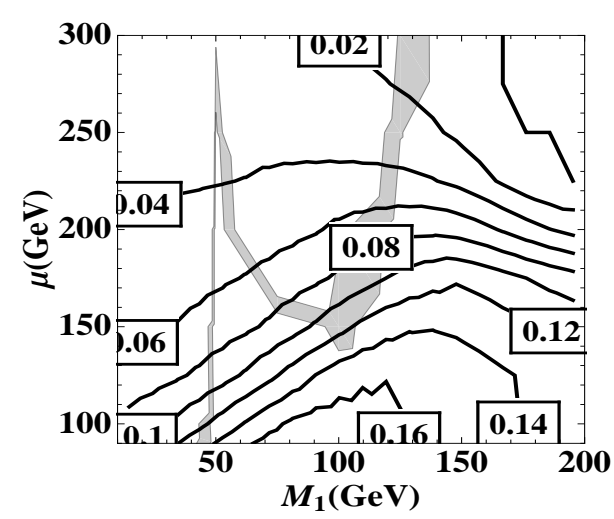

FIG. 9: The contours of constant branching ratio for $\left(H^{0} \rightarrow \tilde{\chi}_{1}^{0} \tilde{\chi}_{1}^{0}\right)$ in the $\mu-M_{1}$ plane for $M_{A}=300 \mathrm{GeV}$ with arbitrary gaugino masses at the GUT scale. Here $M_{2}$ is taken to be $200 \mathrm{GeV}$. The shaded region represents the region allowed by the WMAP data.

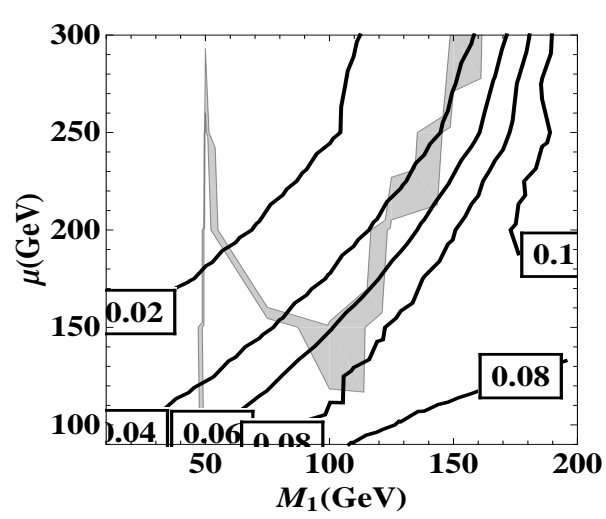

FIG. 10: The contours of constant branching ratio for $\left(H^{0} \rightarrow \tilde{\chi}_{1}^{0} \tilde{\chi}_{1}^{0}\right)$ in the $\mu-M_{1}$ plane for $M_{A}=500 \mathrm{GeV}$ with arbitrary gaugino masses at the GUT scale. Here $M_{2}$ is taken to be $200 \mathrm{GeV}$.

The shaded region is allowed by the WMAP data.

We now proceed to present the results of our calculations. The results are presented as constant branching ratio contours in the $\mu-M_{1}$ plane for two different values of $M_{A} \approx M_{H}=300 \mathrm{GeV}, 500 \mathrm{GeV}$. For the case of arbitrary soft supersymmetry breaking gaugino mass parameters we have taken $M_{2}=200 \mathrm{GeV}$, and $\tan \beta=10$. In Fig. 9 we observe that the branching ratio for $H^{0} \rightarrow \tilde{\chi}_{1}^{0} \tilde{\chi}_{1}^{0}$ can be at most $16 \%$ for $M_{A}=300 \mathrm{GeV}$, and that too in a very narrow region of the parameter space. Furthermore, from Fig. 10 we can see that this branching fraction reduces with the increase in the value of $M_{A}$, having a value of $10 \%$ for $M_{A}=500 \mathrm{GeV}$.

Since we are considering MSSM with $R_{p}$ conservation, the lightest neutralino is an absolutely stable particle. In this case it is important to check that it is not overproduced in the early universe. We have, therefore, calculated the relic density of the lightest neutralino for the range of parameters used in our calculations using micrOMEGAs [57], and imposed the constraints following from the WMAP data [58, 59]. The result of this calculation is shown in Fig.9 as the shaded region allowed by the constraints of the relic density considerations. Similarly, in Fig.10 the shaded region is the region allowed by the WMAP data.

This behavior of the decay $H^{0} \rightarrow \tilde{\chi}_{1}^{0} \tilde{\chi}_{1}^{0}$ may be understood as follows. At low values of $M_{1}, \chi_{i}^{0}$ is bino-like for all values of $\mu$ in the region of the parameter space that we are considering. The Higgs decay to neutralino is suppressed because of small value of $Z_{14}$ for $\tan \beta=10$. The mass of the lightest neutralino increases with the increase in the value of $M_{1}$. In Fig. 9 , for $M_{1} \geq 150 \mathrm{GeV}$ there is a kinematic suppression for $M_{A}=300$ $\mathrm{GeV}$, resulting in a smaller branching ratio. On the other hand for $M_{A}=500 \mathrm{GeV}$ there is no kinematic suppression, hence the branching ratio does not fall off, as is clear from Fig. 10.

For small values of $M_{1}$ and for all values of $\mu$ in the parameter space that we are considering, the quantity $\left(M_{1}-m_{\tilde{\chi}_{1}^{0}}\right)$ is close to zero, so that the decay width for $H \rightarrow \tilde{\chi}_{1}^{0} \tilde{\chi}_{1}^{0}$ is reduced as can be seen from the analytical results in the previous Section, Eq. (IV.3). From Eq. III.10, we observe that $Z_{13}$ is almost zero. Hence the branching fraction for both values of $M_{A}=300 \mathrm{GeV}$ and $500 \mathrm{GeV}$ is small. For fixed $\mu$, with increasing $M_{1}>\mu, \tilde{\chi}_{1}^{0}$ is Higgsino-like, $\left(M_{1}-m_{\tilde{\chi}_{1}^{0}}\right)$ increases, the total width decreases, and hence the branching ratio increases. For fixed $M_{1}$ with decreasing $\mu,\left(M_{1}-m_{\tilde{\chi}_{1}^{0}}\right)$ increases. Furthermore, the denominator(total width) decreases with increasing $\mu$, the numerator decreases faster, and hence the branching fraction decreases with $\mu$. Hence, to obtain a constant branching fraction, we have to increase $M_{1}$ and $\mu$ simultaneously until the factor $\left(1-4 m_{\tilde{\chi}_{1}^{0}}^{2} / M_{H}^{2}\right)^{3 / 2}$ starts dominating, and causes kinematic suppression for the case of $M_{A}=300 \mathrm{GeV}$. 




FIG. 11: The contours of constant branching ratio of $\left(H^{0} \rightarrow \tilde{\chi}_{1}^{0} \tilde{\chi}_{2}^{0}\right)$ in the $\mu-M_{1}$ plane for $M_{A}=300 \mathrm{GeV}$ with arbitrary gaugino masses at the GUT scale. Here $M_{2}$ is taken to be $200 \mathrm{GeV}$. The shaded region is allowed by WMAP data.



FIG. 12: The contours of constant branching ratio for $\left(H^{0} \rightarrow \tilde{\chi}_{1}^{0} \tilde{\chi}_{2}^{0}\right)$ in the $\mu-M_{1}$ plane for $M_{A}=500 \mathrm{GeV}$ with arbitrary gaugino masses at the GUT scale. Here $M_{2}$ is taken to be $200 \mathrm{GeV}$.

The shaded region is allowed by WMAP data.

We note that the total width has been calculated using CalcHEP, and depending on the values of parameters $M_{1}$ and $\mu$ different channels contribute to the total width, which has been used in the calculations presented here.

We may mention here that if we increase the value of $M_{2}>200 \mathrm{GeV}$, large branching fractions for the invisible decay would still be possible. On the other hand, values of $M_{2}<200 \mathrm{GeV}$ will exclude the region of parameter space with considerable invisible branching fraction.

We now turn to the invisible decay $H^{0} \rightarrow \tilde{\chi}_{1}^{0} \tilde{\chi}_{2}^{0}$ for values of $M_{A} \approx M_{H}=300 \mathrm{GeV}$ and $500 \mathrm{GeV}$. In Fig. 11 and Fig. 12 we show the contours of constant branching ratios for this decay. For $M_{A}=300 \mathrm{GeV}$ the branching ratio can at most be $12 \%$ and for $M_{A}=500 \mathrm{GeV}$ it is at most be $6 \%$. As we have seen in case of the decay $H^{0} \rightarrow \chi_{1}^{0} \chi_{1}^{0}$ here also we see a kinematic suppression in case of $300 \mathrm{GeV}$ Higgs boson for large values of $M_{1}$ and $\mu$. The reason is that the sum of the masses of the lightest and the second lightest neutralino reaches its limiting value and the decay $H^{0} \rightarrow \chi_{1}^{0} \chi_{2}^{0}$ is no longer possible. On the contrary, for $500 \mathrm{GeV}$ Higgs boson there is no kinematic suppression, because $M_{H}$ which is nearly equal to $M_{A}$ in the decoupling regime is sufficient to produce a lightest and a second lightest neutralino for this particular region of the parameter space and the decay width is governed by the function $F_{121}$ in Eq.( IV.7). We note that the shaded region in these Figs. represents the region allowed by the WMAP data.

In Fig. 13 and Fig. 14 we show the contours of constant branching ratio for the decay $H^{0} \rightarrow \tilde{\chi}_{2}^{0} \tilde{\chi}_{2}^{0}$ in the $\mu-M_{1}$ plane for values of $M_{A} \approx M_{H}=300$ and $500 \mathrm{GeV}$, respectively. The branching ratio can at most be $2 \%$ in the case of $300 \mathrm{GeV}$ second lightest Higgs, and it reaches a value of about $5 \%$ in case of $500 \mathrm{GeV}$ second lightest Higgs boson. There is a kinematic suppression for $300 \mathrm{GeV}$ Higgs for values of $\mu$ larger than $200 \mathrm{GeV}$ as discussed earlier but for $500 \mathrm{GeV}$ Higgs there is no such suppression and the branching ratio actually increases with increasing $M_{A}$ in some regions of the parameter space. The shaded region in these Figs. represents the region allowed by the WMAP data.

The mass of the second lightest neutralino becomes almost equal to $M_{1}$ for large values of $\mu$ and $M_{1}$. Hence, in this region the branching ratio goes to zero. And from the Fig. 4 we can see that for values larger than $M_{1}=100 \mathrm{GeV}, m_{\tilde{\chi}_{2}^{0}}$ does not depend on $M_{1}$ and depends only on $\mu$. Hence, for a fixed values of $\mu$, the quantities $Z_{22}, Z_{23}$ and $Z_{24}$ increase with $M_{1}$ as $\left(M_{1}-m_{\chi_{1}^{0}}\right)$ increases with $M_{1}$. Therefore, the width $H^{0} \rightarrow \tilde{\chi}_{2}^{0} \tilde{\chi}_{2}^{0}$ also increases. We have checked that the total width decreases with $M_{1}$. So the branching fraction $H^{0} \rightarrow \tilde{\chi}_{2}^{0} \tilde{\chi}_{2}^{0}$ increases with $M_{1}$. Now for a fixed $M_{1}$, the quantity $\left(M_{1}-m_{\chi_{1}^{0}}\right)$ decreases with $\mu$. Furthermore, $Z_{22}$ decreases with $\mu . Z_{23}$ and $Z_{24}$ also decrease, having $\left(M_{1}-m_{\chi_{1}^{0}}\right)$ in the numerator and $\left(m_{\chi_{0}^{2}}+\mu\right.$ sin $\left.2 \beta\right)$ in the denominator. Also, the total width decreases with $\mu$, but the numerator in the branching ratio decreases much faster. Hence the branching fraction $H^{0} \rightarrow \tilde{\chi}_{2}^{0} \tilde{\chi}_{2}^{0}$ decreases with $\mu$. Let us recall the branching ratio increases with $M_{1}$. Consequently we have to increase both $M_{1}$ and $\mu$ to get the contours of constant branching ratio. This is reflected in Figs. 13 and 14. 


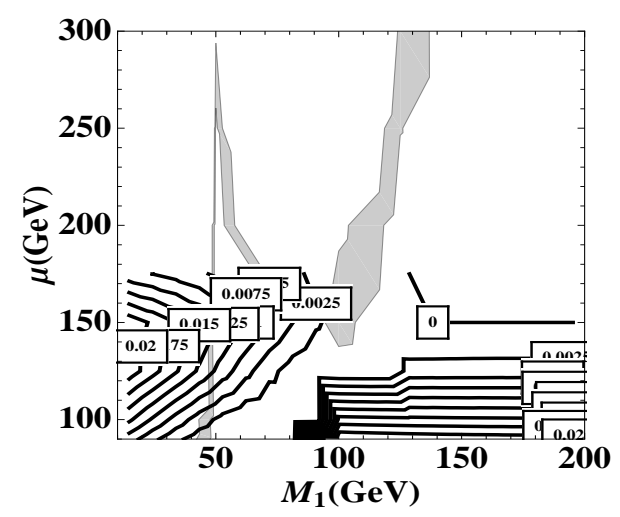

FIG. 13: The contours of constant branching ratio for $\left(H^{0} \rightarrow \tilde{\chi}_{2}^{0} \tilde{\chi}_{2}^{0}\right)$ in the $\mu-M_{1}$ plane for $M_{A}=300 \mathrm{GeV}$ with arbitrary gaugino masses at the GUT scale. Here $M_{2}$ is taken to be $200 \mathrm{GeV}$. The shaded region represents the region allowed by the WMAP data.

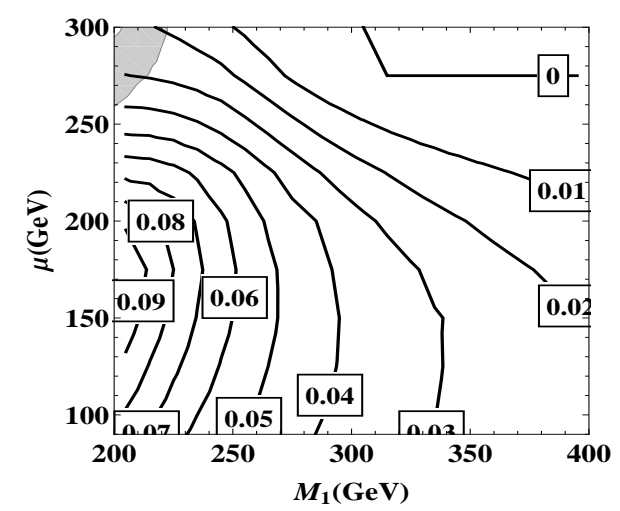

FIG. 15: The contours of constant branching ratio for $\left(H^{0} \rightarrow \tilde{\chi}_{1}^{0} \tilde{\chi}_{1}^{0}\right)$ in the $\mu-M_{1}$ plane for $M_{A}=500 \mathrm{GeV}$ with universal gaugino masses at the GUT scale. The shaded region is allowed by WMAP data.

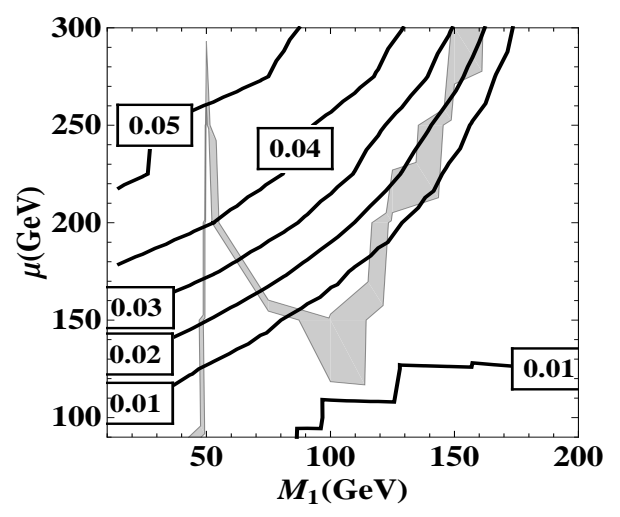

FIG. 14: The contours of constant branching ratio for $\left(H^{0} \rightarrow \tilde{\chi}_{2}^{0} \tilde{\chi}_{2}^{0}\right)$ in the $\mu-M_{1}$ plane for $M_{A}=500 \mathrm{GeV}$ with arbitrary gaugino masses in the $\mu-M_{1}$ plane. Here $M_{2}$ is taken to be 200 $\mathrm{GeV}$. The shaded region is allowed by WMAP data.

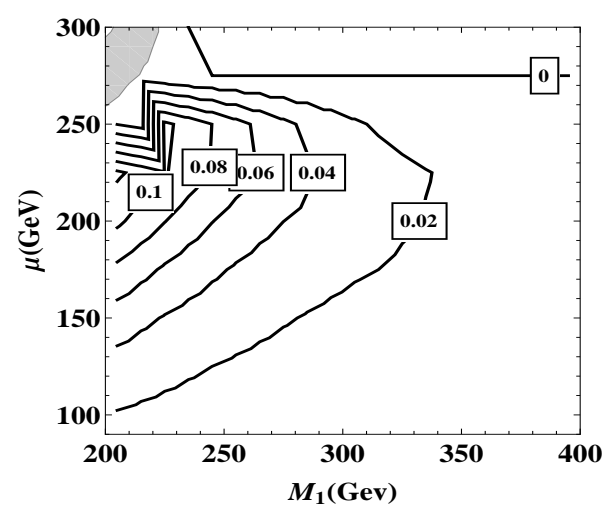

FIG. 16: The contours of constant branching ratio for $\left(H^{0} \rightarrow \tilde{\chi}_{1}^{0} \tilde{\chi}_{2}^{0}\right)$ in the $\mu-M_{1}$ for $M_{A}=500 \mathrm{GeV}$ with universal gaugino masses at the GUT scale. The shaded region is allowed by WMAP data.

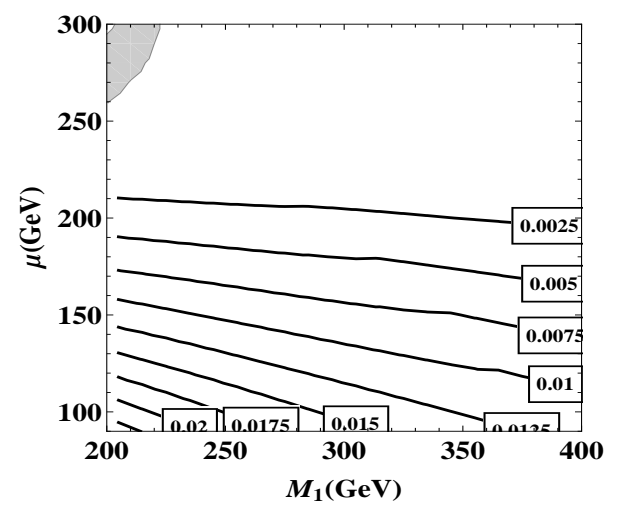

FIG. 17: The contours of constant branching ratio for $\left(H^{0} \rightarrow \tilde{\chi}_{2}^{0} \tilde{\chi}_{2}^{0}\right)$ in the $\mu-M_{1}$ plane for $M_{A}=500 \mathrm{GeV}$ for universal gaugino masses at the GUT scale. The shaded region is allowed by WMAP data. 


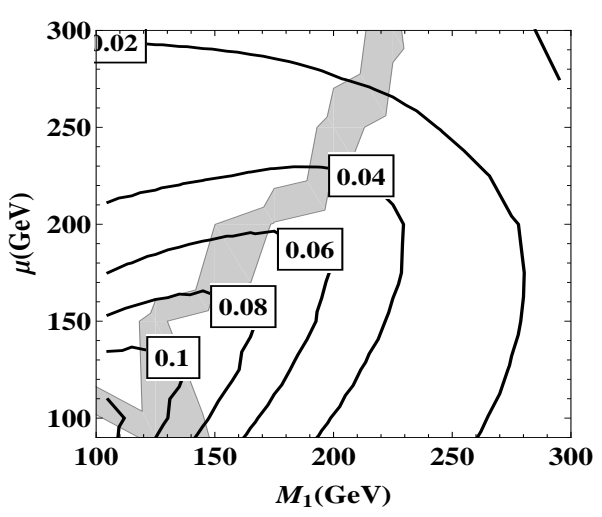

FIG. 18: The contours of constant branching ratio for $\left(H^{0} \rightarrow \tilde{\chi}_{1}^{0} \tilde{\chi}_{1}^{0}\right)$ in the $\mu-M_{1}$ plane for $M_{A}=500 \mathrm{GeV}$ for $\mathrm{SO}(10)$ with non-universal gaugino mass boundary condition. The shaded region is allowed by WMAP data.

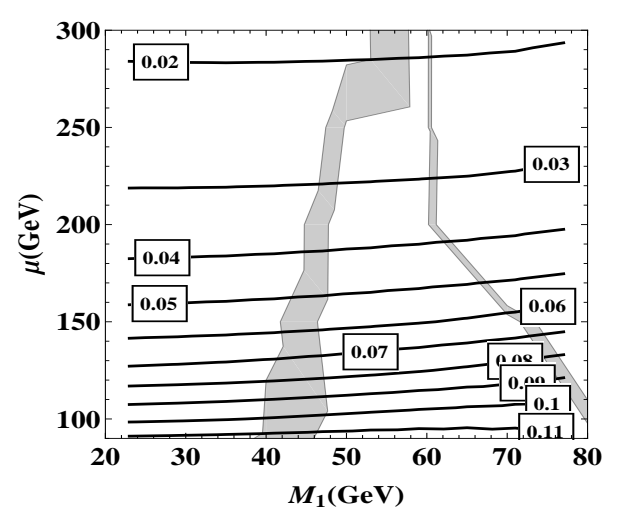

FIG. 19: The contours of constant branching ratio of $\left(H^{0} \rightarrow \tilde{\chi}_{1}^{0} \tilde{\chi}_{1}^{0}\right)$ in the $\mu-M_{1}$ plane for

$M_{A}=500 \mathrm{GeV}$ for $E_{6}$ with non-universal gaugino mass boundary condition. The shaded region is allowed by WMAP data.

Having considered the case of arbitrary gaugino masses at the GUT scale in detail, we now turn to the case of universal boundary conditions. In Figs. 15, 16 and 17 we have plotted the contours of constant branching ratio for $H^{0} \rightarrow \tilde{\chi}_{1}^{0} \tilde{\chi}_{1}^{0}, H^{0} \rightarrow \tilde{\chi}_{1}^{0} \tilde{\chi}_{2}^{0}$, and $H^{0} \rightarrow \tilde{\chi}_{2}^{0} \tilde{\chi}_{2}^{0}$, respectively for a $500 \mathrm{GeV}$ second lightest Higgs boson with universal boundary condition on the gaugino masses at the grand unified scale. In this case the boundary conditions imply $M_{2} \approx 2 M_{1}$ and $M_{3} \approx 7 M_{1}$ at the electroweak scale. Here we can see the invisible branching ratios for $\tilde{\chi}_{1}^{0} \tilde{\chi}_{1}^{0}$ can attain a value $9 \%$ and $\tilde{\chi}_{1}^{0} \tilde{\chi}_{2}^{0}$ can attain a value of $10 \%$. The branching ratio $H^{0} \rightarrow \tilde{\chi}_{2}^{0} \tilde{\chi}_{2}^{0}$ can at most be $2 \%$. We have taken the range of $M_{1}$ to be $200 \mathrm{GeV}$ to $400 \mathrm{GeV}$ for this case. For this case $M_{2}$ and $M_{3}$ depend on $M_{1}$ and to respect the experimental constraints we need to take $M_{3}$ i.e the gluino mass above $1400 \mathrm{GeV}$. As discussed in the previous section for values of $\mu$ larger than $275 \mathrm{GeV}$, the mass of the lightest neutralino exceeds the limiting value of $250 \mathrm{GeV}$. Hence, a $500 \mathrm{GeV}$ Higgs boson cannot decay into lightest neutralino pairs. In the case of the second lightest neutralinos, $\mu=250 \mathrm{GeV}$ is the limiting value because the mass of the second lightest neutralino attains a value of $250 \mathrm{GeV}$ as can be seen from Fig. 6 .

We note from the behavior of the branching ratios that $m_{\tilde{\chi}_{1}^{0}}$ and $m_{\tilde{\chi}_{2}^{0}}$ do not have a significant dependence on $M_{1}$ but depend on $\mu$. This pattern is depicted in Figs. 5 and 6 . The behavior of $H^{0} \rightarrow \tilde{\chi}_{2}^{0} \tilde{\chi}_{2}^{0}$ can also be understood in the same manner. We note that $m_{\tilde{\chi}_{2}^{0}}$ changes linearly with $\mu$ and in large parts of the parameter space it is practically equal to $\mu$. Hence from Eq. III.12 we can see that the width of $H^{0} \rightarrow \tilde{\chi}_{2}^{0} \tilde{\chi}_{2}^{0}$ increases with $M_{1}$. We have checked that the total width decreases with increasing $M_{1}$. Hence the branching fraction also increases with $M_{1}$. The total width decreases with $\mu$. Hence to get constant branching ratio we need increasing values of $M_{1}$ with decreasing values of $\mu$. This can be seen from Fig. 17. The behavior of $H^{0} \rightarrow \tilde{\chi}_{1}^{0} \tilde{\chi}_{1}^{0}$ is also similar to that of $H^{0} \rightarrow \tilde{\chi}_{2}^{0} \tilde{\chi}_{2}^{0}$. The branching fraction is dominated by $\left(M_{1}-m_{\tilde{\chi}_{1}^{0}}\right)$ term which increases with $M_{1}$. We note that for large values of $M_{1}$ the behavior is almost same as that of $H^{0} \rightarrow \tilde{\chi}_{2}^{0} \tilde{\chi}_{2}^{0}$. The shaded region in these Figs. represents the regions allowed by the WMAP data. As we can see from Figs. 15,16 and 17 the universal boundary condition scenario is very much constrained by the WMAP data. Only small regions of the parameter space are allowed which can give rise to some invisible branching ratio.

In Fig. 18 we have considered an example of non-universal boundary condition on the gaugino masses. For this we have chosen a $\mathbf{2 1 0}$ dimensional representation of the gauge group $\mathrm{SO}(10)$ in which $M_{1}: M_{2}: M_{3}$ is in the ratio $1: 6:-14.3$. We have plotted the contours of constant branching ratio for $H^{0} \rightarrow \tilde{\chi}_{1}^{0} \tilde{\chi}_{1}^{0}$ in the $\mu-M_{1}$ plane. We can see that the branching ratio can go upto $10 \%$ for a small region of the parameter space. The shaded region in this Fig. represents the region allowed by the WMAP data.

In Fig. 19 we have considered another example of non-universal boundary condition on gaugino masses for a 2430 dimensional representation of the gauge group $E_{6}$. The gaugino masses are in the ratio $M_{1}: M_{2}: M_{3}$ $=1: 50.2: 70.9$. This particular representation is somewhat special. Because out of all the non-universal boundary conditions this is the only representation in which $\mathrm{r}$ (defined as the ratio $M_{1} / M_{2}$ ) satisfies the the condition $r \leq 0.04$, resulting in a light neutralino. This actually allows the $126 \mathrm{GeV}$ Higgs to decay into lightest neutralinos. In this case we can see the branching ratio for $H^{0} \rightarrow \tilde{\chi}_{1}^{0} \tilde{\chi}_{1}^{0}$ can go upto $11 \%$ for a tiny region of the $\mu-M_{1}$ parameter space. The shaded region in this Fig. represents the region allowed by the WMAP data.

For the case of Fig. 18 we see that for a particular value of $M_{1}$ the branching fraction decreases with $\mu$. Because the quantity $M_{1}-m_{\tilde{\chi}_{1}^{0}}$ decreases with $\mu$ at a particular value of $M_{1}, m_{\tilde{\chi}_{1}^{0}}$ increases with $\mu$ as can be seen 


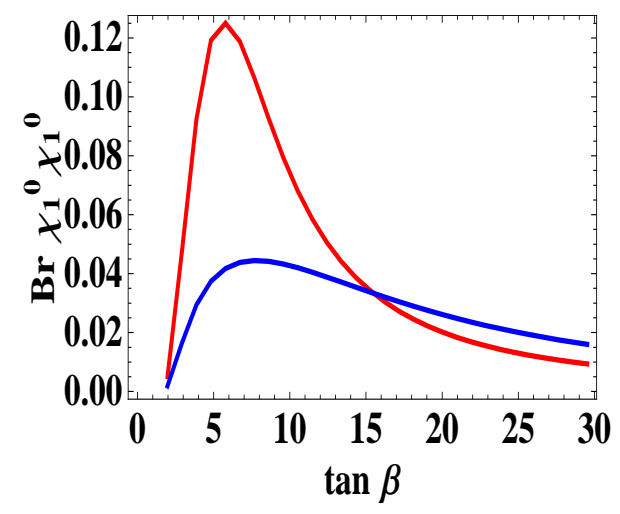

FIG. 20: Branching ratio of $\left(H^{0} \rightarrow \tilde{\chi}_{1}^{0} \tilde{\chi}_{1}^{0}\right)$ with $M_{H}=300 \mathrm{GeV}$ (red), $500 \mathrm{GeV}$ (blue) as a function of $\tan \beta$.



FIG. 21: Branching ratio of $\left(H^{0} \rightarrow \tilde{\chi}_{1}^{0} \tilde{\chi}_{2}^{0}\right)$ with $M_{H}=300 \mathrm{GeV}$ (red), $500 \mathrm{GeV}$ (blue) as a function of $\tan \beta$.

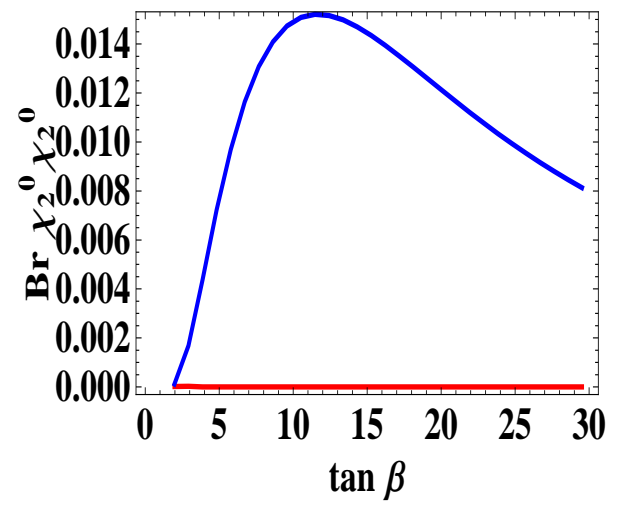

FIG. 22: Branching ratio of $\left(H^{0} \rightarrow \tilde{\chi}_{2}^{0} \tilde{\chi}_{2}^{0}\right)$ with $M_{H}=300 \mathrm{GeV}($ red), $500 \mathrm{GeV}$ (blue) as a function of $\tan \beta$.

from Fig. 7. Hence $Z_{13}$ and $Z_{14}$ decrease with $\mu$. The term $Z_{12}$ also decreases with $\mu$, because the denominator $6 M_{1}-m_{\tilde{\chi}_{1}^{0}}$ decreases with $\mu$, but the rate of decrease is lower than the rate of increase of $M_{1}-m_{\tilde{\chi}_{1}^{0}}$ in the numerator. The total width decreases with $\mu$, but here also the rate of decrease is less, hence the branching fraction decreases with $\mu$ for a particular value of $M_{1}$.

Fig. 19 corresponds to the situation where the mass of the lightest neutralino is almost equal to $M_{1}$ for all $\mu$, as can be seen from Fig. 8. Hence $\left(M_{1}-m_{\tilde{\chi}_{1}^{0}}\right)$ is a very small quantity which is more or less constant as a function of $\mu$. Hence $Z_{12}, Z_{13}$ and $Z_{14}$ all decrease with $M_{1}$ for a particular $\mu$, having $m_{\tilde{\chi}_{1}^{0}}$, which is equal to $M_{1}$, in the denominator. But the total width also decreases with $M_{1}$. Hence the branching fraction remains almost constant as a function of $M_{1}$.

It is known that the $126 \mathrm{GeV}$ Higgs boson of the MSSM can decay invisibly only for arbitrary gaugino masses at the GUT scale. Here we find that for the situation for the second lightest Higgs boson MSSM is far more interesting, because it can decay invisibly, not only for arbitrary gaugino masses but also for constrained boundary conditions of universal and non-universal gaugino masses at the GUT scale.

From the plots we notice that there are kinks in all the contour plots for branching ratios studied in this paper. Although it is difficult to obtain analytical expressions for the constant branching ratio contours, one thing that can be easily understood is that depending on the mass of the neutralinos different decay channels will open up for different range of values of $\mu$ and $M_{1}$. Whenever the total decay width in the denominator changes because of new channels opening up, the constant branching ratio contour plots show kinks.

The range of values of $M_{1}$ and $\mu$ considered in this paper is adequate for our study, because beyond this range of $M_{1}$ and $\mu$ the branching ratios fall and are therefore not relevant.

We have also considered the dependence of the branching ratios for the decays $H^{0} \rightarrow \tilde{\chi}_{1}^{0} \tilde{\chi}_{1}^{0}, H^{0} \rightarrow \tilde{\chi}_{1}^{0} \tilde{\chi}_{2}^{0}$ and $H^{0} \rightarrow \tilde{\chi}_{2}^{0} \tilde{\chi}_{2}^{0}$ on $\tan \beta$. Figs. 20, 21 and 22 show the dependence of these two branching ratios on tan $\beta$ for 


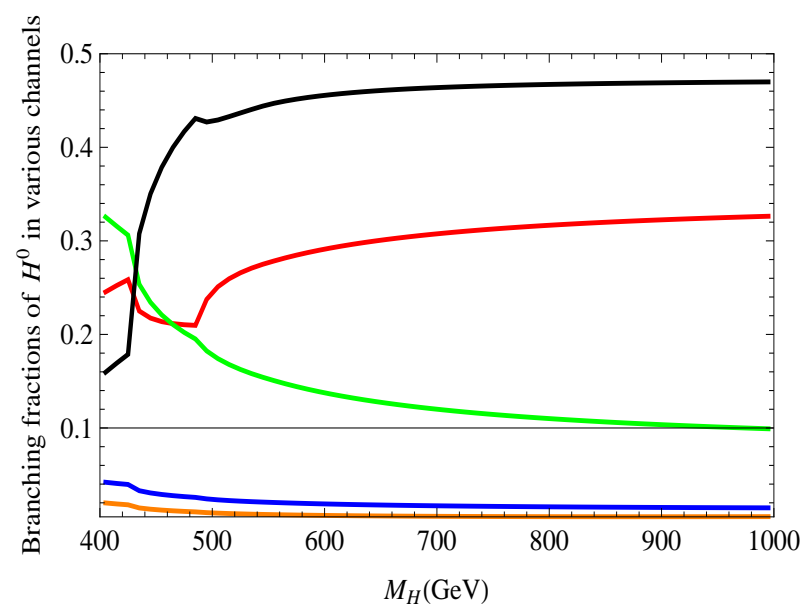

FIG. 23: The branching fraction of the heavier Higgs to $b \bar{b}$ (green), neutralinos(red), charginos(black), $\tau \tau$ (blue), lightest Higgs(orange) as a function of $M_{H}$.

$M_{A} \approx M_{H}=300$ and $500 \mathrm{GeV}$ for the choice of parameters shown in Table III. These values of parameters are consistent with all current experimental constraints.

\begin{tabular}{|c|c|c|c|}
\hline$M_{1}=120 \mathrm{GeV}$ & $M_{S}=1.5 \mathrm{TeV}$ & $M_{A}=300,500 \mathrm{GeV}$ & $M_{2}=200 \mathrm{GeV}$ \\
\hline$M_{3}=1402 \mathrm{GeV}$ & $A_{t}=3600 \mathrm{GeV}$ & $A_{b}=3600 \mathrm{GeV}$ & $\mu=200 \mathrm{GeV}$ \\
\hline
\end{tabular}

TABLE III: Input parameters for the plots of branching ratios versus $\tan \beta$.

The behavior of the $\tan \beta$ dependence of the invisible branching ratios can be understood in the following manner. In MSSM, the $H^{0} \rightarrow b \bar{b}$ coupling is $\frac{\cos \alpha}{\cos \beta}$ times its SM value, and $H^{0} \rightarrow t \bar{t} \operatorname{coupling}$ is $\frac{\sin \alpha}{\sin \beta}$ times the SM value.

At low values of $\tan \beta$ the $H^{0}$ coupling to the up-type quarks is large, hence in this region its decay to top-antitop quarks is dominant, if kinematically allowed. If the second lightest Higgs has a mass of $500 \mathrm{GeV}$, the branching ratio for this decay is $57 \%$. In this region, the decay channel $b \bar{b}$ or the invisible decay channels are not significant. If the Higgs is not so heavy (around $300 \mathrm{GeV}$ ), then top quark channel is kinematically not available, and the main decay channel then will be to two lightest Higgs and some of the invisible channels.

In case of moderate $\tan \beta$, say $\tan \beta=7$, invisible branching ratio for $H^{0} \rightarrow \tilde{\chi}_{1}^{0} \tilde{\chi}_{1}^{0}$ peaks for both $300 \mathrm{GeV}$ as well as 500 Gev Higgs boson.

The branching ratios for $H^{0} \rightarrow \tilde{\chi}_{1}^{0} \tilde{\chi}_{2}^{0}$ also peaks at moderate values of $\tan \beta$ for 300 and $500 \mathrm{GeV}$ second lightest Higgs. On the other hand $\tilde{\chi}_{2}^{0}$ being very heavy, the $300 \mathrm{GeV}$ second lightest Higgs cannot decay into two $\tilde{\chi}_{2}^{0}$ s for any $\tan \beta$ for the parameter space considered. For the $500 \mathrm{GeV}$ second lightest Higgs the branching ratio for $H^{0} \rightarrow \tilde{\chi}_{2}^{0} \tilde{\chi}_{2}^{0}$ peaks at moderate values of $\tan \beta$.

For large values of $\tan \beta \approx 30$, Higgs coupling to the down-type quarks is dominant, and $b \bar{b}$ becomes the dominant decay mode. The invisible decay channels are once again insignificant.

In the case of lightest Higgs decay, in the low $\tan \beta$ region, the invisible decay would be significant as the top decay channel is kinematically closed. In the case of the second lightest Higgs boson, low values of tan $\beta$ do not give significant invisible decay width, but for moderate values of $\tan \beta \approx 10$ the invisible branching ratio can be significant.

In the Fig. 23 we have considered the branching fraction of heavier Higgs boson to all the dominant decay channels. The dominant visible channels are $b \bar{b}, \tau \bar{\tau}, h h[60,61]$. With increase in the values of $M_{H}$, the branching fraction for the $b \bar{b}$ channel decreases, $\tau \bar{\tau}, h h$ channels are not significant, and chargino and neutralino channels become important. Neutralino branching fraction can be as large as 30\%, and chargino branching fraction can be about $50 \%$. For this study the input parameters are $M_{1}=150, M_{2}=200, M_{3}=1400, \mu=200$ and $\tan \beta=10$. From this study we can see that if the second lightest Higgs is heavy enough it can have major decay channels into the electroweak-inos. It can be inferred that in the future LHC run during the direct search for the second lightest Higgs boson, its decay into supersymmetric particles may play an important role in the decoupling regime. 


\section{A. "Quasi-invisible" decays}

We would like to draw attention of the reader to the fact that all the decay channels of the second lightest Higgs boson that we have considered and studied in detail in this paper are not truly invisible. $H^{0} \rightarrow \tilde{\chi}_{1}^{0} \tilde{\chi}_{1}^{0}$ channel is truly invisible as the lightest neutralino $\tilde{\chi}_{1}^{0}$ is the lightest supersymmetric particle. It is the only stable supersymmetric particle assuming conservation of R-parity. All other heavy neutralinos will decay into the LSP and SM particles. Hence $H^{0} \rightarrow \tilde{\chi}_{1}^{0} \tilde{\chi}_{2}^{0}$ and $H^{0} \rightarrow \tilde{\chi}_{2}^{0} \tilde{\chi}_{2}^{0}$ cannot be considered invisible. In ref [62] the authors have extensively studied the electrowikino decays for different regions of the parameter space of the electroweak sector without assuming any SUSY-breaking mediation scenario. There is a region of the parameter space defined as scenario $\mathrm{C}$ in the paper where $|\mu|<M_{1}, M_{2}$. In this region of the parameter space $\tilde{\chi}_{1}^{0}$ and $\tilde{\chi}_{2}^{0}$ are both Higgsino like and their masses are almost equal to the Higgsino mass $\mu$ and hence they are almost mass-degenerate. It has been pointed out in the same reference that the LSP multiplet production will be difficult to observe at the LHC because of the mass degeneracy and the soft decay products especially when the production is suppressed. Hence one has to be extremely careful while looking at the decay of the electrowikinos at the future LHC. Where the $\tilde{\chi}_{1}^{0}$ and $\tilde{\chi}_{2}^{0}$ are almost mass degenerate, we have termed this kind of decays $H^{0} \rightarrow \tilde{\chi}_{1}^{0} \tilde{\chi}_{2}^{0}$ and $H^{0} \rightarrow \tilde{\chi}_{2}^{0} \tilde{\chi}_{2}^{0}$ as 'quasi-invisible' decay. It is worth mentioning that, one must expect that the International Linear Collider(ILC) will be able to identify the soft decay products, leptons and jets of mass $10 \mathrm{GeV}$ or less, which are produced in the mass-degenerate case, because ILC has a cleaner environment for event reconstruction [62].

Another intriguing possibility that could render some of these channels "visible" is to adapt a strategy that has been used in the past for dark matter searches. In other words a promising search for invisible decays is in the monojet channel as in the case for dark matter search at the LHC. When the second lightest Higgs boson decays into invisible particles, monojet searches involving initial state radiation from the gluons that fuse to produce the Higgs boson could be used in these channels so the the Higgs decaying into light neutralinos does not necessarily escape the detection at the colliders. This same procedure is used to look for dark matter at the LHC. In case of invisible decays one has to look for monojet signals $H^{0} \rightarrow \tilde{\chi}_{1}^{0} \tilde{\chi}_{1}^{0}+j e t$ as $\tilde{\chi}_{1}^{0}$ is the lightest supersymmetric particle. In ref [63-65] it has been shown that the monojet signature carries a good potential to constrain the invisible decay width of $\mathrm{a} \approx 125 \mathrm{GeV}$ Higgs boson in a model independent fashion using the monojet search results by ATLAS and CMS [66, 67]. Now in case of MSSM with two CP-even Higgs, we can employ a different strategy while looking for the second lightest Higgs boson. One can calculate the production cross section $\times$ invisible branching in the MSSM for specific regions of the parameter space. There are several Standard Model processes which can act as background for the monojet signals. $p p \rightarrow Z(\rightarrow \nu \bar{\nu})+j e t$ is the main irreducible background with the same topology as our signal. There is QCD background as well. The backgrounds can be estimated [68]. The background can be reduced significantly using several $p_{T}$ cuts on the jet and missing transverse energy cut. Then one can find the dependence of signal significance $\mathcal{S}=N_{S} / \sqrt{N_{B}}\left(N_{S}\right.$ is the number of signal events and $N_{B}$ is the number of background events) on different parameters for $14 \mathrm{TeV}$ HLLHC with the desired integrated luminosity $\mathcal{L}$. One can use the LHC monojet search results at $14 \mathrm{TeV}$, ie. the limits on the monojet events to probe the regions in the MSSM parameter space spanned by $M_{A}$, tan $\beta, \mu$ [69]. Thus one can exclude regions of the MSSM parameter space at a desired signal significance(90\% or $95 \%$ C.L).

\section{DISCUSSION AND CONCLUSION}

In this work we have considered the possibility of invisible decays of the second lightest $\operatorname{Higgs} \operatorname{boson}\left(H^{0}\right)$ in the MSSM in the decoupling regime. In the past, various studies have shown that certain regions of the parameter space of MSSM, allow a Higgs boson in the mass range 123-129 GeV both in the decoupling and non-decoupling regime, satisfying the LHC constraints. For most of the parameter space, the lightest Higgs decay to the lightest neutralinos is kinematically allowed, leading to invisible decay modes. The main objective of those works was to prove that it would therefore be very important to study the couplings of the newly discovered particle at high precision. Global fits have been performed on the couplings of the newly discovered particle, in order to place upper bounds on the invisible decay width. Taking into account these bounds, the parameter space of these new physics scenarios can be further constrained, since the regions giving a large invisible Higgs decay branching ratio will be in conflict with the experiments.

In the present work, which is a sequel to previous investigations [18], we are looking at the problem from a different point of view. We are asking a related question. We have considered the intriguing possibility that the heavier CP even Higgs $\left(H^{0}\right)$ of the MSSM would decay into invisible LSP's. Knowing the invisible branching ratios of the second lightest Higgs boson one can look for it in the future HL-LHC or ILC through the monojet signals. From the limits given by the colliders on the monojet signal one can then probe the MSSM parameter space at a desired confidence level. Hence it will also be possible to detect or rule out the second lightest 
Higgs boson in the MSSM in a certain mass range from the monojet searches for its invisible decay. In other words, in our previous paper [18], the study has been done to constrain the MSSM parameter space looking at the possible invisible decay width of the newly discovered $126 \mathrm{GeV}$ Higgs boson. But in this work we are following a different route and trying to make a theoretical prediction on the search for the second lightest Higgs boson of the MSSM. Our point is to understand the invisible decay width of $H^{0}$ consistent with the current experimental constraints. In the scenarios that we considered earlier, which partly motivated the present study, we had considered that the $125 \mathrm{GeV}$ Higgs boson had some partial decay channels into light neutralinos in the MSSM, or that it would decay into neutralinos or light CP odd Higgs particles which could be present in the spectrum of the NMSSM. In [25] the intriguing possibility was considered that the $125 \mathrm{GeV}$ Higgs was not that lightest CP even Higgs but the heavier one, while the lightest evaded detection altogether. Here, we have more conservative assumptions, viz., that the $125 \mathrm{GeV}$ resonance is indeed the lightest CP even Higgs, but consider the possibility that the heavier one has invisible decays. This scenario can arise naturally in the decoupling region, i.e., the second lightest Higgs boson is quite heavy and could be produced at future experiments at the LHC which will eventually decay into lighter particles. These final states include neutralinos, charginos, $b b, Z Z, \tau \tau, h h$. In the present work we have tried to carry out an exhaustive search of the MSSM parameter space where the second lightest Higgs boson can decay into the lightest neutralinos and therefore remains invisible. We have considered the decay of the second lightest Higgs into the second lightest neutralinos also, because there are certain regions of the parameter space where the lightest and the second lightest neutralino are almost mass-degenerate and hence the second lightest neutralino may remain invisible('quasi-invisible') at the LHC. We have used semi-analytical formulas as a guide to our study. In the decoupling regime, which is a realistic scenario, the values of $M_{A}$ and $M_{H}$ are nearly equal.

For our parameter choices guided by recent constraints by LEP and LHC, we have taken the gaugino masses at the electroweak scale to be within present constraints. We have chosen $A_{t}$ judiciously to get the lightest Higgs mass in the range in which it has been detected. We have taken soft SUSY breaking scale $M_{S}$ to large, around $1.5 \mathrm{TeV}$ consistently. In this paper, we have presented a detailed analysis first of the Higgs sector as well as that of the neutralino sector, in order to isolate the conditons under which it becomes kinematically feasible for $\left(H^{0}\right)$ to decay invisibly. We have considered three different scenarios and have scanned the parameter space in terms of invisible decay of the second lightest Higgs boson in these three scenarios, namely arbitrary, universal and non-universal boundary condition on the soft gaugino masses at the GUT scale. Our analysis reveals that it is typically not possible to have such regions in the MSSM with GUT scale universal boundary conditions on the soft gaugino masses. This can be seen from Figs. 15, 16 and 17. The situation remains similar even with non-universal boundary conditions as can be seen from Figs. 18 and 19. The main reason for this is that there is not sufficient freedom in the choice of the gaugino masses with these boundary conditions. On the other hand, relaxing all constraints, as is the case with general (arbitrary) boundary conditions, allows the invisible branching ratios to be considerable. This can be seen from Figs. 9, 10, 11, 12, 13 and 14.

From our study we conclude that there is a significant portion of the parameter space where the invisible decays can be quite significant. For instance, with universal or non-universal boundary conditions, the invisible branching ratio is not enhanced, but if we relax this constraint we can have more significant branching ratio in invisible decays. Hence the monojet searches for the invisible decay of the second lightest Higgs boson at the future colliders can be used as a probe to look for the second lightest Higgs boson as well as to put constraints on the regions of the MSSM parameter space. This would be most useful in the case of arbitrary boundary condition on the gaugino masses at the GUT scale. One has to be more careful and find different strategies in the case of universal and non-universal boundary condition.

\section{ACKNOWLEDGEMENTS}

P.N.P would like to thank Inter-University Centre for Astronomy and Astrophysics, Pune, where part of this work was done, for hospitality. The work of P.N.P is supported by the Raja Ramanna Fellowship of the Department of Atomic Energy, and partly by the J. C. Bose National Fellowship of the Department of Science and Technology, India. J.L would like to thank Dr. Biplob Bhattacherjee and Dr. Monalisa Patra for useful and enlightening discussions. B.A would like to thank Professor Daniel Wyler and the University of Zurich, where part of this work was done, for hospitality .

[1] [ATLAS Collaboration], Phys. Lett. B 716, 1 (2012) [arXiv:1207.7214 [hep-ex]].

[2] [ATLAS Collaboration], ATLAS-CONF-2013-014. 
[3] [CMS Collaboration], Phys. Lett. B 716, 30 (2012) [arXiv:1207.7235 [hep-ex]].

[4] S. Chatrchyan et al. [ CMS Collaboration], arXiv:1303.4571 [hep-ex].

[5] J. Wess and J. Bagger, "Supersymmetry and supergravity," Princeton, USA: Univ. Pr. (1992) 259 p.

[6] H. P. Nilles, Phys. Rept. 110, 1 (1984); P. Nath, R. L. Arnowitt and A. H. Chamseddine, NUB-2613.

[7] P. Fayet, Nucl. Phys. B 90, 104 (1975).

[8] H. P. Nilles, M. Srednicki and D. Wyler, Phys. Lett. B 120, 346 (1983).

[9] J. R. Ellis, J. F. Gunion, H. E. Haber, L. Roszkowski and F. Zwirner, Phys. Rev. D 39, 844 (1989).

[10] M. Drees, Int. J. Mod. Phys. A 4, 3635 (1989).

[11] P. N. Pandita, Phys. Lett. B 318, 338 (1993).

[12] P. N. Pandita, Z. Phys. C 59, 575 (1993).

[13] U. Ellwanger, Phys. Lett. B 303, 271 (1993) [hep-ph/9302224].

[14] T. Elliott, S. F. King and P. L. White, Phys. Lett. B 314, 56 (1993) [hep-ph/9305282].

[15] P. N. Pandita Phys. Rev. D 50, 571 (1994).

[16] P. N. Pandita, Z. Phys. C 63, 659 (1994).

[17] S. Y. Choi, J. Kalinowski, G. A. Moortgat-Pick and P. M. Zerwas, Eur. Phys. J. C 22, 563 (2001) [Addendum-ibid. C 23, 769 (2002)] [arXiv:hep-ph/0108117].

[18] B. Ananthanarayan, J. Lahiri, P. N. Pandita and M. Patra, Phys. Rev. D 87, 115021 (2013) [arXiv:1306.1291 [hep-ph]].

[19] G. Belanger, B. Dumont, U. Ellwanger, J. F. Gunion and S. Kraml, Phys. Rev. D 88, 075008 (2013) $[\operatorname{arXiv}[1306.2941]]$.

[20] A. Djouadi and G. Moreau, Eur. Phys. J. C73 (2013) 9, 2512 [arXiv[1303.6591]].

[21] G. Aad et al. [ATLAS Collaboration], [arXiv:1402.3244 [hep-ex]] .

[22] [ATLAS Collaboration], ATLAS-CONF-2013-011.

[23] [CMS Collaboration], Search for invisible Higgs produced in association with a Z boson, CMS-PAS-HIG-13-018

[24] [CMS Collaboration], Search for invisible Higgs decays in the VBF channel, CMS-PAS-HIG-13-013

[25] P. N. Pandita and M. Patra, Phys. Rev. D 89, 115010 (2014) [arXiv:1405.7163 [hep-ph]].

[26] Tong Li, Phys. Lett. B 728, 77-84 (2014)[arXiv:1309.6713 [hep-ph]].

[27] P. N. Pandita, Phys. Lett. B 151, 51 (1985).

[28] S. Heinemeyer, O. Stal, G. Weiglein, Phys. Lett. B 710 (2012) 201 [arXiv:1112.3026 [hep-ph]].

[29] E. Boos, A. Djouadi, M. Muhlleitner, A. Vologdin, Phys. Rev. D 66, 055004 (2002) [hep-ph/0205160].

[30] E. Boos, A. Djouadi, A. Nikitenko, Phys. Lett. B 578, 384 (2004) [hep-ph/0307079].

[31] N. D. Christensen T. Han, S. Su, Phys. Rev. D 85, 115018 (2012) [arXiv:1203.3207 [hep-ph]].

[32] H E. Haber, [arXiv:hep-ph/9501320]

[33] A. Djouadi, L. Maiani, G. Moreau, A. Polosa, J. Quevillon, V. Riquer, Eur. Phys. J.C (2013) 73:2650 [arXiv:1307.5205 [hep-ph]]

[34] L. Maiani, A. D. Polosa and V. Riquer, Phys. Lett. B 718, 465 (2012) [arXiv:1209.4816 [hep-ph]].

[35] A. Belyaev, N. D. Christensen and A. Pukhov, arXiv:1207.6082 [hep-ph].

[36] L. Maiani, A. D. Polosa, V. Riquer, New. J. Phys. 14 (2012) 073029 [arXiv:1202.5998 [hep-ph]]

[37] L. Maiani, A. D. Polosa, V. Riquer, Phys. Lett. B718 (2012) 465 [arXiv:1209.4816 [hep-ph]]

[38] A. Djouadi, J. Quevillon, JHEP 1310 (2013) 028 [arXiv:1304.1787 [hep-ph]]

[39] A. Bartl, H. Fraas, W. Majerotto and N. Oshimo, Phys. Rev. D 40, 1594 (1989).

[40] H. E. Haber and G. L. Kane, Phys. Rept. 117, 75 (1985).

[41] M. Guchait, Z. Phys. C 57, 157-163 (1993).

[42] S. P. Martin and P. Ramond, Phys. Rev. D 48, 5365 (1993) [hep-ph/9306314].

[43] S. Schael et al. [ALEPH and DELPHI and L3 and OPAL and SLD and LEP Electroweak Working Group and SLD Electroweak Group and SLD Heavy Flavour Group Collaborations], Phys. Rept. 427, 257 (2006) [hep-ex/0509008].

[44] E. Cremmer, S. Ferrara, L. Girardello and A. Van Proeyen, Phys. Lett. B 116, 231 (1982).

[45] I. Gogoladze, J. D. Lykken, C. Macesanu and S. Nandi, Phys. Rev. D 68, 073004 (2003) [hep-ph/0211391].

[46] G. Bertone, D. Hooper and J. Silk, Phys. Rept. 405, 279 (2005) [hep-ph/0404175].

[47] S. Heinemeyer, W. Hollik, A. M. Weber and G. Weiglein, JHEP 0804, 039 (2008) [arXiv:0710.2972 [hep-ph]].

[48] [ATLAS Collaboration], ATLAS-CONF-2012-033.

[49] [CMS Collaboration], CMS-PAS-SUS-12-005.

[50] H. K. Dreiner, J. S. Kim and O. Lebedev, Phys. Lett. B 715, 199 (2012) [arXiv:1206.3096 [hep-ph]].

[51] K. Griest and H. E. Haber, Phys. Rev. D 37, 719 (1988).

[52] John F. Gunion, H E. Haber, Phys. Rev. D 37, 2515 (1988).

[53] M. Carena and H. E. Haber, [arXiv-hep-ph/0208209]

[54] Talk by A. Hoecker at the Lepton-Photon Conference, Stanford, June 2013

[55] CMS Collaboration, JHEP 10(2014) 160, [arXiv:1408.3316]

[56] A. Arbey, M. Battaglia, A. Djouadi, F. Mahmoudi, JHEP 1209 (2012) 107 [arXiv:1207.1348]

[57] G. Bélanger, F. Boudjema, A. Pukhov and A. Semenov, Comput. Phys. Commun. 192, 322 (2015) [arXiv:1407.6129 [hep-ph]].

[58] C. Bennett et al. [WMAP Collaboration], Astrophys. J. Suppl. 148, 97 (2003) [astro-ph/0302208]

[59] D. N. Spergel et al. [WMAP Collaboration], Astrophys. J. Suppl. 148, 175 (2003) [astro-ph/0302209].

[60] CMS Collaboration,CERN/LHCC 2006-021, CMS TDR 8.2, 26 June 2006

[61] M. Carena and H. E. Haber, I. Low, N R. Shah, C. E. M. Wagner, Phys. Rev. D91(2015) 3, 035005, [arXiv:1410-4969] 
[62] Tao Han, Sanjay Padhi, Shufang Su, Phys.Rev.D88(2013)11,115010, [arXiv:1309.5966]

[63] A. Djouadi, A. Falkowski, Y. Mambrini,J. Quevillon, Eur. Phys. J.C 73, 2455(2013), [arXiv:1205.3169]

[64] Y. bai, P. Draper,J. Shelton, JHEP 1207,192(2012),[arXiv:1112.4496]

[65] C. Englert, J. Jaeckel, E. Re, M. Spannowsky, Phys.Rev.D 85, 035008(2012), [arXiv:1111.1719]

[66] The CMS Collaboration, JHEP 09 (2012) 094, arXiv:1206.5663[hep-ex]

[67] The ATLAS Collaboration, ATLAS-CONF-2012-147

[68] C. Han et.al, JHEP 1402(2014)049,[arxiv:1310.4274]

[69] B. Bhattacharjee, A. Chakraborty, A. Choudhury, [arXiv:1504:04308] 